Max-Planck-Institut für demografische Forschung

Max Planck Institute for Demographic Research

Konrad-Zuse-Strasse 1 - D-18057 Rostock · GERMANY

Tel +49 (0) 3812081 - 0; Fax +49 (0) 3812081 - 202;

http://www.demogr.mpg.de

MPIDR WORKING PAPER WP 2005-025

AUGUST 2005

\title{
Season of birth influences life span of Mediterranean fruit flies, rats and mice
}

\author{
Anna V. Semenchenko (semenchenko@gmx.net) \\ Gabriele Doblhammer (doblhammer@ demogr.mpg.de) \\ Vladimir N. Anisimov \\ James Carey \\ Anatoli I. Yashin
}

This working paper has been approved for release by: James W. Vaupel (jwv@ demogr.mpg.de)

Head of the Laboratory of Survival and Longevity.

(C) Copyright is held by the authors.

Working papers of the Max Planck Institute for Demographic Research receive only limited review. Views or opinions expressed in working papers are attributable to the authors and do not necessarily reflect those of the Institute. 


\title{
Season of birth influences life span of Mediterranean fruit flies, rats and mice
}

\author{
Anna V. Semenchenko ${ }^{1, * \dagger}$, Gabriele Doblhammer ${ }^{1}$, Vladimir N. Anisimov², \\ James Carey $^{3,4}$ and Anatoli I. Yashin ${ }^{5}$ \\ ${ }^{1}$ Max Planck Institute for Demographic Research, Konrad-Zuse-Strasse 1, 1805\%, Rostock, \\ Germany \\ ${ }^{2}$ N.N.Petrov Research Institute of Oncology, St.Petersburg, Russia \\ ${ }^{3}$ Department of Entomology, University of Callifornia, Davis, CA 95616, USA \\ ${ }^{4}$ Center for the Economics and Demography of Aging, University of California, Berkeley, CA \\ 94720, USA \\ ${ }^{5}$ Center for Demographic Studies, Duke University, Durham NC, USA
}

Keywords: Frailty model, laboratory animals, mortality, seasonality, survival patterns

\section{Introduction}

Recent research shows that the month of birth influences life span and disease in humans (Doblhammer, 2004). Nutrition of the mother during pregnancy and/or virus infections inutero or in the first few months of life have been proposed as underlying causal mechanisms. In this study we ask the question whether the season-of-birth effect in life span also exists in other species than humans and in particular, whether it exists in laboratory animals that are used as control groups in experiments. These animals live under strictly controlled environmental conditions and should not be subject to seasonal changing dietary conditions and exposure to virus infections. We use data from large experiments on Mediterranean fruit flies with thousands of animals, as well as smaller experiments on rats and mice. In all three species we find a significant season-of-birth pattern in life span that is not only consistent among the species but also shares similar characteristics with the pattern observed among humans.

\section{Material and Methods}

\subsection{General idea}

The original idea was to check the data available from experiments on different laboratory animals for the presence of the month-of-birth effect on survival. Revision of available data on rodents and fruit flies with known month of birth/eclosion revealed the facts that: (i) the number of animals in some month-of-birth groups is insufficient for analysis (e.g. mice); (ii) the data for some months are missing (e.g. rats); (iii) the difference in life span distributions between two consecutive months is sometimes not significant (e.g. rats, medflies).

We decided to consolidate month-of-birth groups according to four seasons: winter December, January and February, spring - March, April and May, summer - June, July and August, and autumn - September, October and November. Such a manipulation not only increases the number of animals in groups, but also allows us to assume that gestation period (2-3 weeks of developmental period in pupae stage for medflies, 18-21 days of pregnancy for mice and 21-23 days - for rats) falls in the same season as birth/eclosion. It is important because seasonal differences in environmental conditions during gestation period may be the cause of differences in survival of adults. The period of breast feeding for mammals is also

\footnotetext{
${ }^{*}$ Author for correspondence (semenchenko@gmx.net)

${ }^{\dagger}$ Current address: N.N.Petrov Research Institute of Oncology, St.Petersburg, Russia
} 
important, but because it is short for observed rodents (weaning age is 21-28 days for mice and 21 days for rats) we can assume it to be in the same season as birth.

For simplicity's sake, for all laboratory animals considered, we used the winter-born/eclosed group as a control one and compared all other season-of-birth groups to it.

In spite of the assumption that laboratory conditions are constant and highly controlled over the year, it should be noticed that there are seasonal changes in weather conditions on both sites, where experiments were conducted. In St. Petersburg, Russia, (elevation: 20 meters, latitude: $5955 \mathrm{~N}$, longitude: $03015 \mathrm{E}$ ) monthly average temperature changes from $-6^{\circ} \mathrm{C}$ in winter (the lowest in January with average variation from $-9^{\circ} \mathrm{C}$ to $-4^{\circ} \mathrm{C}$ ) to $+15^{\circ} \mathrm{C}$ in summer (the highest in June with variation from $+13^{\circ} \mathrm{C}$ to $+21^{\circ} \mathrm{C}$ ). Most recorded rainfalls $(16-20 \mathrm{~cm})$ are in period from June to September, average morning relative humidity $(82-90 \%)$ is higher than $86 \%$ (yearly average) from July to December, average evening relative humidity (51-85\%) is higher than 70\% (yearly average) from October to February. In Tapachula, Mexico, (elevation: 108 meters, latitude: $1454 \mathrm{~N}$, longitude: $09215 \mathrm{~W}$ ) monthly average temperature is almost constant during the year: $+24^{\circ} \mathrm{C}-+27^{\circ} \mathrm{C}$ with variation from $+17^{\circ} \mathrm{C}$ to $+34^{\circ} \mathrm{C}$. Average precipitation is the highest in May-October and it is between $28 \mathrm{~cm}$ (May) and $46 \mathrm{~cm}$ (September). Average relative humidity (66-82\%) is greater than $75 \%$ (yearly average) from May to November.

\subsection{Laboratory animals}

\section{Mediterranean fruit flies (Ceratitis capitata)}

Five different experiments were conducted from 1993 to 1996 aiming to investigate the influence of different types of caloric restriction on survival of Mediterranean fruit flies (medflies) Ceratitis

capitata. Studies were conducted at the Moscamed medfly mass rearing facility in Metapa (near Tapachula), Chiapas, Mexico. Adult medflies of both sexes were maintained in mesh-covered, $15 \times 60 \times 90 \mathrm{~cm}$ aluminum cages under the following environmental conditions: 12:12 light-dark cycle, $24^{\circ} \mathrm{C}( \pm 2)$ and $65 \%$ relative humidity $( \pm 9 \%)$. For every experimental cohort (dietary restricted) a control cohort (fed a full diet of protein, sugar and water ad libitum), emerged at the same date, was observed. Aiming to investigate season-of-eclosion influence on life span, we used the data only from the control cohorts for analysis.

\section{Mice}

Female CBA mice were purchased from the Rappolovo Animal Farm of the Russian Academy of Medical Sciences by the N.N. Petrov Research Institute of Oncology (St. Petersburg, Russia) for drug testing experiments. Mice were kept 5 per polypropylene cages $(30 \times 21 \times 10 \mathrm{~cm})$ under standard light/dark regimen (12 hrs light, $12 \mathrm{hrs}$ dark) at a temperature of $22 \pm 2{ }^{\circ} \mathrm{C}$ and received standard laboratory chow (Anisimov et al., 2003) and tap water ad libitum. We used information about the life span of 82 animals with known month of birth, that served as control groups in different experiments in 1998-2002.

\section{Rats}

We analyzed the life span of 294 male and 276 female outbred Wistar-derived LIO rats (Anisimov et al., 1989) with known month of birth, that were bred at the Animal Department of the N.N. Petrov Research Institute of Oncology (St. Petersburg, Russia). These animals served as intact control groups in different drug testing experiments in 1968-1973 and were kept in polypropylene cages $(38,5 \times 28,5 \times 14,5 \mathrm{~cm}), 6$ rats per cage at a temperature of $22 \pm 2^{\circ} \mathrm{C}$. A regimen of $12 \mathrm{hrs}$ of light followed by $12 \mathrm{hrs}$ of dark was maintained. The animals received standard laboratory chow (Baranova et al., 1985) and tap water ad libitum. 


\section{$2.3 \quad$ Statistical methods}

\section{Empirical estimates of survival functions}

For each group empirical estimates of mortality rates at the age of $j$ days were calculated using the ratio

$$
q_{j}=\frac{d_{j}}{n_{j-1}}
$$

where $d_{j}$ is the number of dead animals observed during the $j$-th day of life and $n_{j-1}$ is the number of animals alive at the end of the previous day. The Kaplan-Meier estimates of experimental conditional survival functions (Kalbfleisch and Prentice, 1980), given the animals survived the age $x^{*}$, were calculated as the cumulative product:

$$
S_{j}=\prod_{i=x^{*}}^{j}\left(1-q_{i}\right) .
$$

Minimal life span observed in population of animals of the same sex was used as $x^{*}$.

\section{Comparison of the life span distributions and relative risks of death}

The log-rank test statistic (Cox and Oakes, 1988) and two-sample Kolmogorov-Smirnov twosided test were used to test the null hypothesis that there is no difference in the life span distributions of the populations eclosed/born in different seasons.

For every experimental group the Cox's regression model (Cox, 1972) was used to estimate relative risk of death for the groups born in different seasons compared to the winter-born group, which served as a control for other seasons:

$$
\mu_{k}(t, z)=\mu_{0}(t) \exp \left(z \beta_{k}\right), \quad k=1,2,3,
$$

where $k$ codes for season ( 1 - spring, 2 - summer, 3 - autumn), $\mu_{k}(t, z)$ and $\mu_{0}(t)$ denote the conditional hazard and baseline hazard rates, respectively, $\beta_{k}$ is the unknown parameter for corresponding season-of-birth group, and $z$ takes values 0 and 1 , being an indicator variable for two samples - the control (winter-born) and the group of interest (other than winter season of birth), respectively.

\section{Testing heterogeneity assumption}

To investigate whether the data on survival of laboratory animals born in different seasons may be described by a discrete heterogeneity model (Yashin et al., 2002), we assumed that each population consists of two subgroups called "weak" and "robust". Let $p_{w}$ denotes the proportion of weak individuals in the population. Then proportion of robust animals $p_{r}$ is equal to $1-p_{w}$. We assumed that mortality rates in these subgroups are ordered; that is, mortality in the weak subgroup is higher than mortality in the robust one. The survival function in the population is a mixture of two survival functions:

$$
S(x)=p_{w} S_{w}(x)+\left(1-p_{w}\right) S_{r}(x) .
$$

This model allows us to estimate proportion of weak individuals in the population as well as survival functions in both subgroups. We assume that survival functions for the weak and robust subgroups correspond to the Gompertz mortality curves: $\mu_{w / r}(x)=a_{w / r} e^{\beta_{w / r} x}$ (Gompertz, 1825), which were, however, taken to be different for different seasons. We applied this model only to the populations of rats in order to separate the weak subgroups from the populations. 
Analysis of heterogeneity in mortality in populations of flies, mice and robust subgroups of rats was also conducted using semi-parametric model of heterogeneous mortality, which allows comparison of several groups of interest to one control group.

The idea behind the heterogeneous mortality model (also called frailty model) is that for every individual in the population the risk of death is proportional to the unobserved characteristic called frailty or heterogeneity variable: $\mu(x, z)=z \mu_{0}(x)$, where $z$ denotes frailty and $\mu_{0}(x)$ is a baseline hazard, which is, generally, not observed.

The risk of death for the individual whose frailty is equal to 1 corresponds to the baseline hazard. Given fixed frailty, the baseline hazard reflects intensity of damage occurrence in the individual organism due to environmental conditions and aging: better conditions - lower level of the baseline hazard, slow aging - slow growth rate of the baseline hazard. Given the baseline hazard, frailty can be considered as a factor that reflects the ability of an organism to withstand harsh environmental conditions: lower frailty - better protection of an organism more robust individual.

The assumption about stratification of the population into several frailty classes ("weak", "robust", etc.) is replaced by more realistic assumption of continuously distributed heterogeneity variable. We assumed frailty to be Gamma-distributed with mean 1 and variance $\sigma^{2}$ (as in Vaupel et al., 1979) and compared survival functions of groups born in different seasons to the one of the winter-born group in terms of differences in the baseline hazard and frailty distribution. Detailed description of the model and derivation of its semi-parametric representation are given in the Appendix.

Denoting conditional survival function (given $x \geq x^{*}$ ) for the control group as $S_{c}$ and survival functions for other groups as $S_{k}, k=1,2,3$, we can write an expression for the survival in season of interest as follows (See the Appendix):

$$
S_{k}(x)=\left(1+r_{k} \gamma_{k}\left(S_{c}(x)^{-\sigma^{2}}-1\right)+\gamma_{k} r_{k} \sigma^{2} \frac{\alpha_{k}}{\beta_{k}}\left(e^{\beta_{k}\left(x-x^{*}\right)}-1\right)\right)^{-\frac{1}{\gamma_{k} \sigma^{2}}} .
$$

This representation (we call it semi-parametric) allows us to avoid the widely-used but biologically unjustified assumption of a parametric form for the baseline hazard, because observed survival function in winter-born group $S_{c}(x)$ is used in the representation of $S_{k}(x)$.

The model has four unknown parameters $\alpha_{k}, \beta_{k}, r_{k}, \gamma_{k}$ that are specific to each season of eclosion/birth and one parameter $\sigma^{2}$ that is common to all groups - the frailty variance in the winter-born group.

Differences in the baseline hazard are modeled as an additive function of age and controlled by parameters $\alpha$ and $\beta$. If $\beta=0$, changes in parameter $\alpha$ reflect permanent (constant with age) decrease or increase of the baseline hazard in the group of interest compared to the control group, depending on whether $\alpha$ is greater or less than zero. We attribute such differences to changes in environmental conditions with season. Parameter $\beta$ describes the amplification or disappearance of the $\alpha$-effect with age, according to whether $\beta$ is greater or less than zero.

Parameter $r \neq 1$, being a ratio of means of the frailty distributions in the population of interest and the control group (See the Appendix), shows relative difference in the average frailty between these groups. $r<1$ reflects the situation when group of interest is more robust on average than the control one. When $r>1$, the average frailty of the group of interest is greater than one of the control group. Parameter $\gamma$ describes non-linear transformation of individuals frailties and reflects the differences in the variance of the frailty distributions in the control group and the population of interest. $\gamma \neq 1$ indicates an increase $(\gamma>1)$ or decrease $(\gamma<1)$ in the frailty variance that corresponds to increase or decrease of the population heterogeneity. As we defined frailty to be the characteristic of an organism to withstand harsh environmental conditions, changes in individual's frailty reflect the ability of an organism to switch on additional defense or reparation mechanisms in order to reduce damages produced by unfavorable environment. 
To compare survival functions for groups born/eclosed in spring-autumn to the wintereclosed group, three specifications of the model were considered. The first one deals only with the differences in the average frailties of the populations $(\alpha=0, r \neq 1, \gamma=1)$. In the second, differences in the mean of the frailty distributions are accompanied by differences in the baseline hazards $(\alpha \neq 0, \beta=0, r \neq 1, \gamma=1)$. The third specification describes differences in survival patterns between groups of interest and the control group as a combination of differences in the baseline hazard and both parameters of the frailty distribution $(\alpha \neq 0, \beta \neq 0, r \neq 1, \gamma \neq 1)$. Because these specifications of the model are nested, the likelihood ratio statistics was used to determine which one gives the best fit to the data.

\section{Parameters estimation procedure}

To obtain the estimates of the model parameters for each experimental data set (flies, mice and rats), the observations of life spans in all season-of-birth groups were used simultaneously. The maximum likelihood approach was implemented and parameters were estimated using a nonlinear optimization procedure (Fletcher, 1987).

Because the structure of the data from medflies experiments corresponds to the number of dead and alive insects during discrete time periods, log-likelihood function is derived from the binomial distribution, where binomial probabilities $q_{j}$ depend on model parameters:

$$
\log \operatorname{Lik}=\sum_{k=1}^{3} \sum_{j}\left(m_{k, j} \ln \left(q_{k, j}\right)+\left(n_{k, j}-m_{k, j}\right) \ln \left(1-q_{k, j}\right)\right),
$$

where $k$ codes for season (spring, summer, autumn), $m_{k, j}$ is the number of deaths on day $j$ of life in the group eclosed in $k$ season, and $n_{k, j}$ is the number of individuals in the same group, which were alive on day $j-1$. Values $q_{k, j}$ are related to survival functions for the groups of interest by the relationship:

$$
q_{k, j}=1-\frac{S_{k}(j+1)}{S_{k}(j)} .
$$

Log-Likelihood function for mice and rats data sets has form:

$$
\operatorname{LogLik}=\sum_{k=1}^{3} \sum_{x_{i, k}} f_{k}\left(\alpha_{k}, \beta_{k}, r_{k}, \gamma_{k}, \sigma^{2}, x_{i, k}\right),
$$

where $k$ codes for season, $x_{i, k}$ is observed life span of the $i$-th animal from $k$-th group, $f_{k}\left(\alpha_{k}, \beta_{k}, r_{k}, \gamma_{k}, \sigma^{2}, x_{i, k}\right)$ - density function of survival distribution in each season-of-birth group. By definition, $f(\cdot, \cdot)=\mu(\cdot, \cdot) S(\cdot, \cdot)$, expressions for mortality $\mu$ and survival function $S$ are given in the Appendix (equations (9) and (10))

Confidence intervals for parameter estimates were calculated using the bootstrap method (Davison and Hinkley, 1997).

\section{Results}

\subsection{Medflies}

Basic statistics on life span of medflies eclosed in different seasons are given in Table 1. Female flies eclosed in winter and male flies eclosed in either winter or autumn lived on average longer than insects eclosed in other seasons. Mean life span of females is longer than the one of males in all season, except autumn. This is also true for the long-lived flies (the last $10 \%$ of survivors).

As can be seen in Figure 1, seasonal changes in average life span form U-shaped pattern and look very similar for males and females (especially long-lived ones), with the lowest values 
Table 1: Life span characteristics of male and female medflies eclosed in different seasons

\begin{tabular}{lllllllll}
\hline & \multicolumn{4}{c}{ males } & \multicolumn{5}{c}{ females } \\
& winter & spring & summer & autumn & winter & spring & summer & autumn \\
\hline Nb. & 45437 & 109837 & 206463 & 46677 & 41992 & 100436 & 187316 & 45491 \\
mean ls & 16.29 & 13.64 & 13.57 & 16.93 & 18.07 & 14.91 & 14.50 & 14.82 \\
se & 0.04 & 0.02 & 0.02 & 0.04 & 0.04 & 0.02 & 0.02 & 0.04 \\
std & 7.89 & 6.88 & 7.32 & 8.07 & 8.63 & 7.30 & 7.65 & 7.97 \\
mean ls (10\%) & 31.04 & 27.23 & 28.23 & 33.45 & 33.89 & 29.11 & 30.02 & 31.90 \\
se & 0.07 & 0.04 & 0.04 & 0.08 & 0.06 & 0.05 & 0.04 & 0.10 \\
age 90\% & 26 & 22 & 23 & 27 & 29 & 24 & 25 & 25 \\
\hline
\end{tabular}

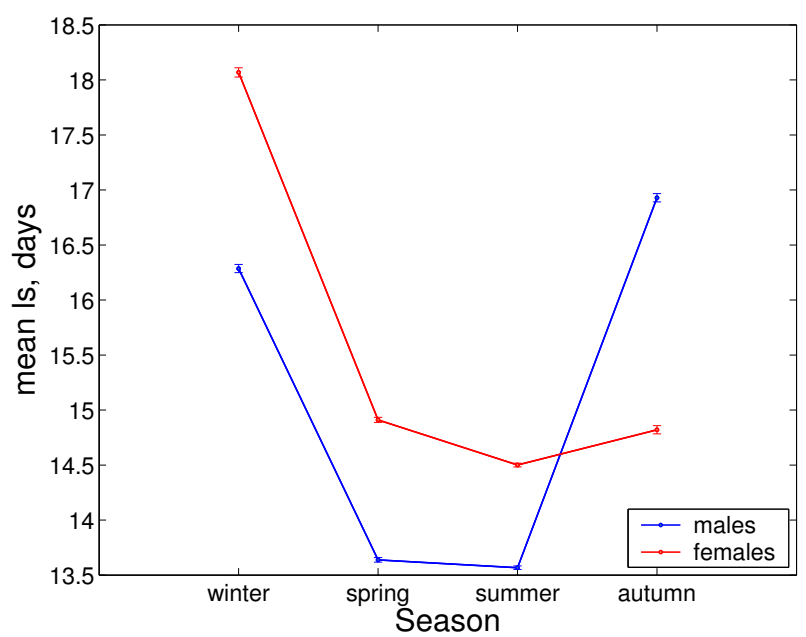

a. all flies

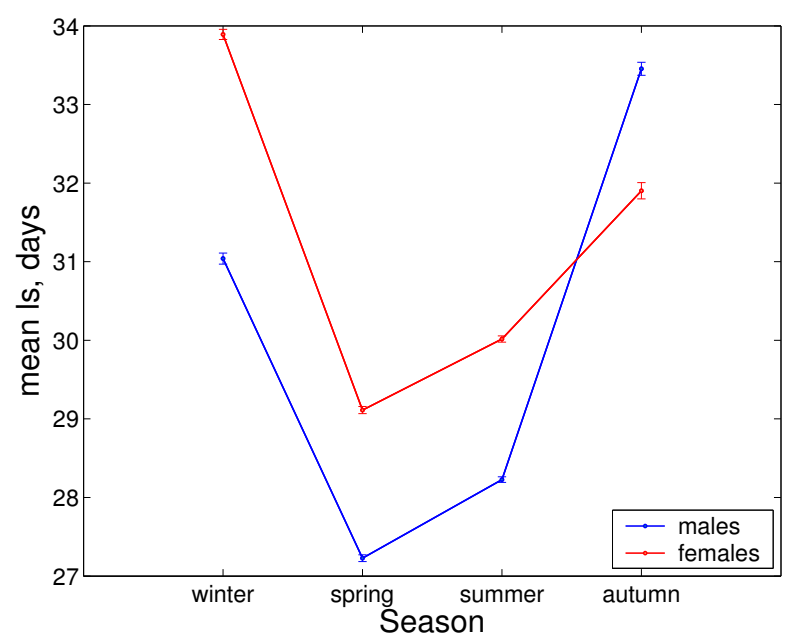

b. last $10 \%$

Figure 1: Changes in life span of medflies with season of eclosion.

in spring and summer. The maximal absolute difference in the average life span of flies is 3.36 days for males and 3.57 for females. It reaches 6.22 and 4.78 days for long-lived males and females, respectively.

According to the log-rank and two-sample Kolmogorov-Smirnov tests the difference in observed life span distributions is significant for different seasons in male and female medflies $(p<0.0001)$, except for females eclosed in spring and autumn. Empirical probabilities of death for the groups of medflies eclosed in different seasons are presented in Figure 2. It can be seen, that both male and female flies eclosed in spring and summer have higher chances of dying in almost all ages, than the flies eclosed in winter. As for those eclosed in autumn, males have lower probabilities of death compared to the winter-eclosed group, especially at young and old ages (before 10 and after 22 days of life). Female flies eclosed in autumn have higher chances of survival than the winter-eclosed group before the age of about 6 days. After this age the empirical probabilities of death for the autumn-eclosed group are very close to those emerged in spring and summer. After the age of about 21 days, the autumn-eclosed female flies have lower probabilities of dying than the spring- and summer-eclosed flies, and after the age of about 25 days probability of death for the autumn-eclosed group becomes even lower than for the winter-eclosed population.

According to the Cox's proportional hazard model, the risks of death among male medflies eclosed in spring and summer are significantly higher than of those eclosed in winter $\left(e^{\beta}=1.43\right.$ 


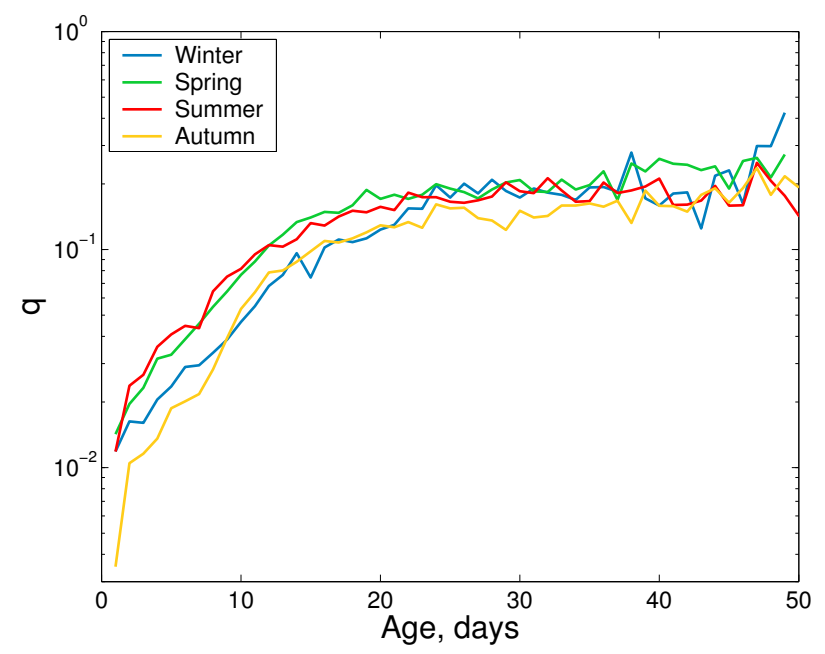

a. males

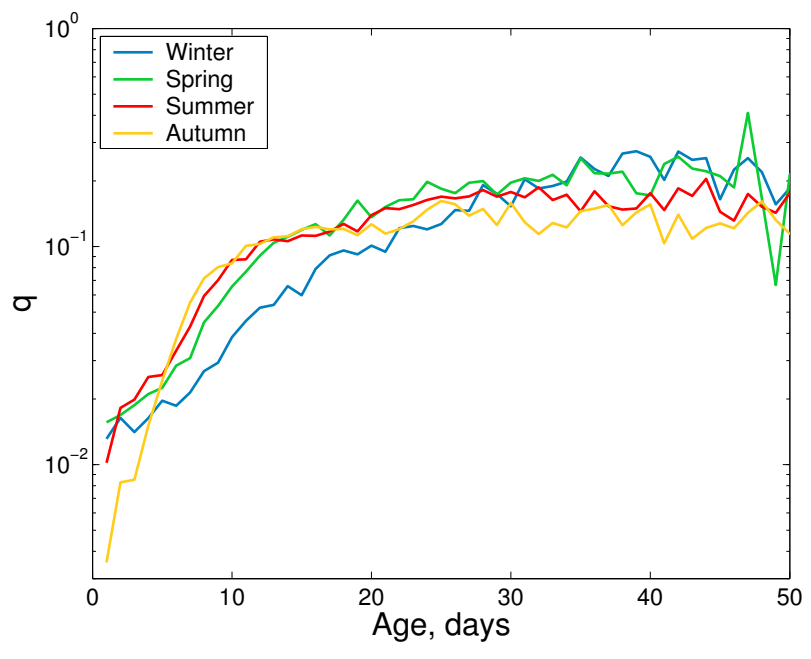

b. females

Figure 2: Empirical probabilities of death (log scale) for medflies eclosed in different seasons.

and $e^{\beta}=1.38$, respectively, $p<0.0001$ ). Male medflies eclosed in autumn have slightly lower risk of dying $\left(e^{\beta}=0.94, p<0.0001\right)$ compared to the flies eclosed in winter. The flies eclosed in summer have lower risk of death than the flies eclosed in spring $\left(e^{\beta}=0.98, p=0.0004\right)$. For the female flies, eclosion in the season other than winter means an increase in the risk of death up to $50 \%$ (relative risks are 1.51, 1.5, and 1.45 for spring, summer, and autumn, respectively, $p<0.0001$ in all cases).

According to the likelihood ratio statistics, the specification of the semi-parametric model of heterogeneous mortality, which takes into account differences in the baseline hazard and both parameters of the frailty distribution, fits the data better than two others (respective $p$-values for the models $r, \sigma^{2}$ and $\alpha, r, \sigma^{2}$ are 0.0001 and 0.0002). Estimated values of the model parameters are presented in Table 2. Fit of the model to empirical survival functions is shown in Figure 3.

Table 2: Estimated parameters of the semi-parametric heterogeneous mortality model for survival of medflies eclosed in different seasons.

\begin{tabular}{|c|c|c|c|}
\hline & \multicolumn{3}{|c|}{ season of eclosion } \\
\hline & spring & summer & autumn \\
\hline \multicolumn{4}{|c|}{ male medflies } \\
\hline$\alpha$ & $-0.0051(-0.0052 ;-0.005)$ & $0.024(0.023 ; 0.025)$ & $-0.009(-0.01 ;-0.009)$ \\
\hline$\beta$ & $-0.12(-0.125 ;-0.12)$ & $0.25(0.249 ; 0.25)$ & $-0.17(-0.17 ;-0.165)$ \\
\hline$r$ & $1.8(1.79 ; 1.8)$ & $0.46(0.45 ; 0.461)$ & $1.03(1.03 ; 1.033)$ \\
\hline$\gamma$ & $1.3(1.28 ; 1.3)$ & $4.72(4.71 ; 4.73)$ & $1.39(1.38 ; 1.4)$ \\
\hline$\sigma^{2}$ & \multicolumn{3}{|c|}{$0.2635(0.2621 ; 0.2647)$ for all seasons } \\
\hline \multicolumn{4}{|c|}{ female medflies } \\
\hline$\alpha$ & $0.0053(0.0052 ; 0.0054)$ & $0.024\left(\begin{array}{lll}0.023 & 0.024\end{array}\right)$ & $0.016(0.015 ; 0.016)$ \\
\hline$\beta$ & $0.31(0.31 ; 0.32)$ & $0.31(0.3 ; 0.31)$ & $0.55(0.54 ; 0.56)$ \\
\hline$r$ & $0.73(0.73 ; 0.74)$ & $0.32(0.31 ; 0.33)$ & $0.15(0.14 ; 0.15)$ \\
\hline$\gamma$ & $5.1(5.04 ; 5.13)$ & $6.4(6.3 ; 6.5)$ & $14.3(14.2 ; 14.5)$ \\
\hline$\sigma^{2}$ & \multicolumn{3}{|c|}{$0.2926(0.2921 ; 0.2936)$ for all seasons } \\
\hline
\end{tabular}

It can be seen (Table 2) that the frailty variance in the winter-eclosed group of females is 


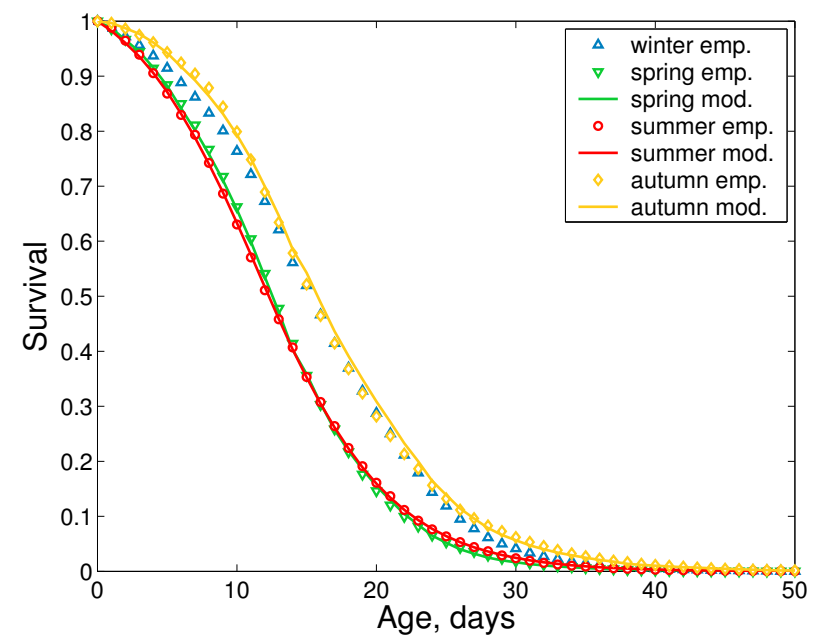

a. males

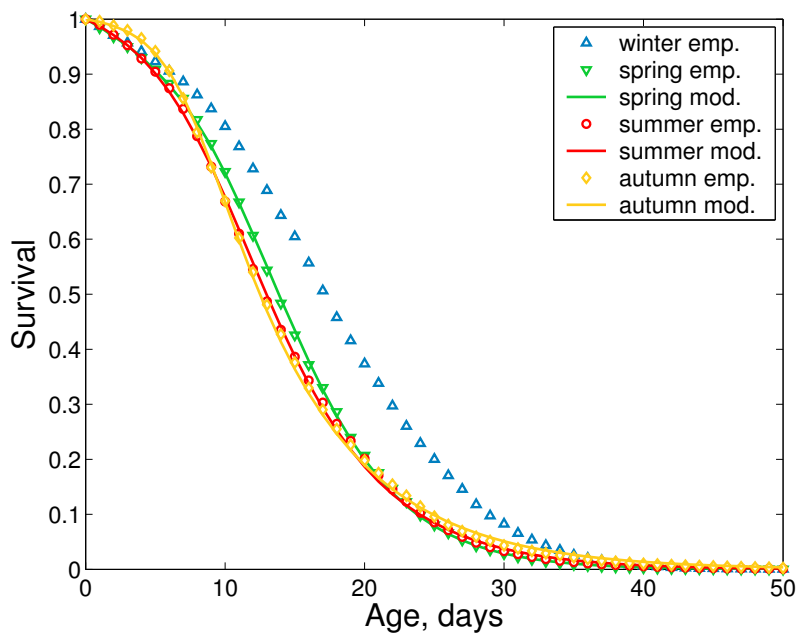

b. females

Figure 3: Empirical and modeled survival functions for medflies eclosed in different seasons.

slightly, however significantly, greater than the one of the male group (parameter $\sigma^{2}$ ).

Although male flies eclosed in spring and summer have almost equal chances of survival in all ages (Fig. 2.a and 3.a), the proposed model, due to its flexibility, enables us to explain the difference in survival patterns in these two groups. Compared to the winter-eclosed population, the spring-eclosed group has a deceased baseline hazard at younger ages $(\alpha<0$ and $\beta<0$, see Tab. 2), and the summer-eclosed group has an increased baseline hazard in all ages $(\alpha>0$ and $\beta>0)$. This difference contributes to a tiny improvement in survival of the spring-eclosed population, compared to the summer-eclosed one. At the same time, the spring-eclosed group is more frail $(r>1)$ and the summer-eclosed group is more robust on average $(r<1)$. This explains why the improvement in survival of the spring-eclosed group is so small and observed only at young ages. Both spring and summer-eclosed groups are more heterogeneous $(\gamma>1)$ than the winter-eclosed population, and the frailty variance in the summer group is the greatest among all groups of male medflies. This huge increase in heterogeneity of summer-eclosed group ensures the long tail of survival function and higher survival values at the old ages, than demonstrated by group eclosed in spring. The baseline hazard of the autumn-eclosed male medflies is lower in young ages $(\alpha<0$ and $\beta<0)$, and this population is almost as robust on average as the winter-eclosed one $(r \approx 1)$, but also more heterogeneous $(\gamma>1)$. This combination of effects results in improvement of survival at young and old ages of the population eclosed in autumn, compared to the winter-eclosed and other groups.

As for female medflies, eclosion during a season other than winter increases the baseline hazard $(\alpha>0$ and $\beta>0)$, average robustness $(r<1)$ and heterogeneity $(\gamma>1)$ of the population, compared to eclosion in winter. Robustness and heterogeneity of the population increase monotonically from spring to autumn. With an increase of heterogeneity, probabilities of death at the old ages become lower (Fig. 2.b) and the tail of survival function becomes thicker (Fig. 3.b). Amplified with age increase of the baseline hazard reduces survival probabilities for flies eclosed in spring-autumn. The rates of amplification $(\beta>0)$ are almost identical in the groups eclosed in spring and summer, but initial increase of the baseline hazard is greater in the summer-eclosed group, that ensures lower survival values of the latter. Relatively small (compared to the summer-eclosed group) initial values of increase of the baseline hazard $(\alpha>0)$ and the greatest increase of the average robustness $(r<1)$ in the autumn-eclosed population result in the highest values of survival at young ages. The drop of survival, which is observed later, is due to the rapid amplification of increase of the baseline hazard $(\beta>0)$ in this group. 


\subsection{Mice}

Basic statistics on life span of mice born in different seasons is presented in Table 3. Despite relatively small number of animals in each season-of-birth group, some significant effects in longevity can be detected. The shortest average life span is inherent to the mice born in summer. The longest average life span, as well as minimal and maximal, was observed in the winter-born group. The average life span of the spring-born mice is lower, however insignificantly, from the one of the winter-born mice. The average life span of autumn-born mice is shorter than the one of the spring-born (insignificant) and winter-born animals.

Table 3: Changes in life span of mice by season of birth

\begin{tabular}{lllll}
\hline & winter & spring & summer & autumn \\
\hline & females & & & \\
Nb. & 15 & 13 & 30 & 24 \\
Mean ls & 319.2 & 302.62 & 281.2 & 289.25 \\
Se & 16.13 & 13.45 & 8.12 & 9.32 \\
Min & 247 & 224 & 223 & 190 \\
Max & 431 & 376 & 391 & 360 \\
Mean ls (25\%) & 399.25 & 359.33 & 336.13 & 340.83 \\
Se & 11.02 & 8.33 & 12.0 & 14.55 \\
Age 75\% & 380 & 336 & 303 & 325 \\
\hline
\end{tabular}

Seasonal changes in the mean life span of mice, also of long-lived quarter of the population, are presented in Figure 4. These changes correspond to a declining pattern with slight increase in the autumn. The maximal absolute difference in average life span between seasons is 38 days and for long lived animals - 63.12 days.

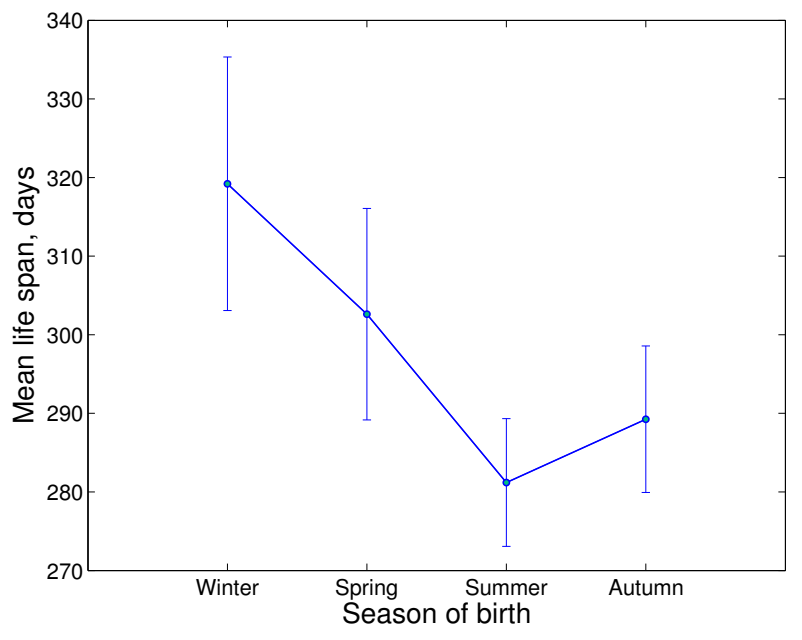

a. all

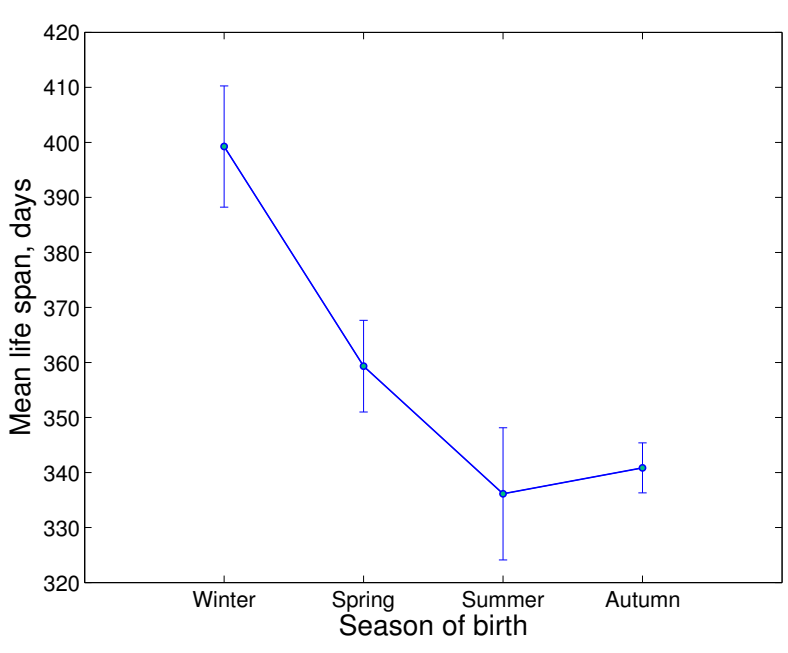

b. last $25 \%$

Figure 4: Mean life span of mice grouped by season of birth.

According to the log-rank test, life span distributions of mice born in different seasons are different $(p=0.04)$ in spite of small number of animals in each group. The pairwise difference is significant between groups born in summer and autumn, compared to the mice born in winter $(p=0.02$ and $p=0.05)$. For female mice, being born in summer or autumn doubles risk of death (according to the Cox's model, $e^{\beta}=2.23$ with $p=0.03$ and $e^{\beta}=2.2$ with $p=0.05$, respectively). 
The specification of the semi-parametric heterogeneous mortality model, which takes into account differences in both baseline hazard and frailty distribution, fits the data better than two others (respective $p$-values for the models $r, \sigma^{2}$ and $\alpha, r, \sigma^{2}$ are 0.003 and 0.007 ). Parameter estimates of this specification are given in the Table 4 . The fit of the model to the empirical survival functions is shown in Figure 5.

Table 4: Estimated parameters of the semi-parametric heterogeneous mortality model for survival of female mice born in different seasons.

\begin{tabular}{llll}
\hline & & \multicolumn{2}{c}{ season of birth } \\
& spring & summer & autumn \\
\hline$\alpha$ & $1.515(1.51 ; 1.52)$ & $0.137(0.133 ; 0.139)$ & $4.017(4.0 ; 4.034)$ \\
$\beta$ & $0.023(0.021 ; 0.024)$ & $0.047(0.043 ; 0.048)$ & $0.025(0.023 ; 0.027)$ \\
$r$ & $5.62 \mathrm{e}-3(5.1 \mathrm{e}-3 ; 5.8 \mathrm{e}-3)$ & $4.73 \mathrm{e}-2(4.1 \mathrm{e}-2 ; 4.9 \mathrm{e}-2)$ & $2.67 \mathrm{e}-2(2.51 \mathrm{e}-2 ; 2.84 \mathrm{e}-2)$ \\
$\gamma$ & $0.095(0.093 ; 0.098)$ & $10.19(10.181 ; 10.21)$ & $0.011(0.009 ; 0.012)$ \\
$\sigma^{2}$ & \multicolumn{3}{c}{$0.1547(0.1543 ; 0.1551)$ for all groups } \\
\hline
\end{tabular}

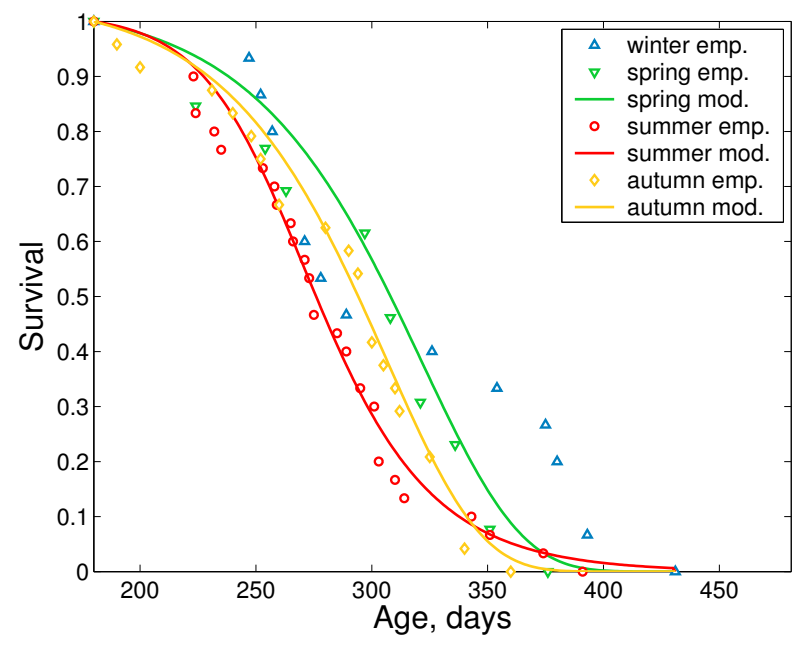

Figure 5: Empirical and modeled survivals

It can be seen (Figure 5) that the chances of survival in winter born group are considerably higher than in the other groups at the young and old ages. The summer-born group has the lowest chances of survival at almost all ages, however the 'tail' of survival function is quite long. Autumn-born mice started to die and the whole group died out earlier than animals from other groups, however survival on the age interval form 270 to 370 days is improved, compared to the summer-born group.

The baseline hazard for mice born in seasons other than winter was estimated as increased $(\alpha>0)$. These populations are also more robust on average $(r<1)$, compared to the winterborn group. Spring and autumn-born groups are more homogeneous $(\gamma<1)$ and the summerborn groups is much more heterogeneous $(\gamma>1)$ than the population born in winter. Although initial increase in the baseline hazard for the groups of mice born in spring and autumn is higher $(\alpha>0)$, the amplification of this effect with age is more rapid in the summer-born group $(\beta>1)$. Together with lower level of increased robustness $(r<1)$ it determines lower survival chances for this group, compared to others. Mice born in autumn demonstrated higher survival values compared to the spring-born group because of lower level of the baseline hazard, higher level of robustness and heterogeneity. The highest frailty variance $(\gamma>1)$ in the population 
of the summer-born mice ensures the longest maximal life span observed among all animals (except those born in winter).

\section{$3.3 \quad$ Rats}

Basic statistics on life span in groups of male and female rats born in different seasons (except spring) are given in the Table 5. Rats, both males and females, born in winter lived on average

Table 5: Changes in life span of rats by season of birth

\begin{tabular}{lllllll}
\hline & males & & \multicolumn{3}{c}{ females } \\
& winter & summer & autumn & winter & summer & autumn \\
\hline Nb. & 86 & 89 & 119 & 92 & 66 & 114 \\
Mean ls & 778.03 & 590.19 & 462.96 & 655.7 & 560.23 & 517.06 \\
6.21 & 27.95 & 33.95 & 23.6 & & & \\
Min & 352 & 90 & 53 & 102 & 92 & 33 \\
Max & 1427 & 1097 & 1027 & 1089 & 880 & 1065 \\
Mean ls $(25 \%)$ & 1063.86 & 941.09 & 822.4 & 919.91 & 844.24 & 799.41 \\
Se & 23.16 & 13.02 & 19 & 12.92 & 5.46 & 17.73 \\
Age $75 \%$ & 912 & 845 & 680 & 851 & 812 & 694 \\
\hline
\end{tabular}

longer then ones born in summer or autumn. This is also true for the quantile of long-lived individuals (the last $25 \%$ of survivors). Estimated mean life span for male rats born in winter is significantly longer than for females. Female rats born in autumn lived on average longer than males. As for long-lived animals, their average life spans are longer among winter and summer-born males compared to those of females. Seasonal changes in the life span of male and female rats are presented in Figure 6 and correspond to a declining pattern.

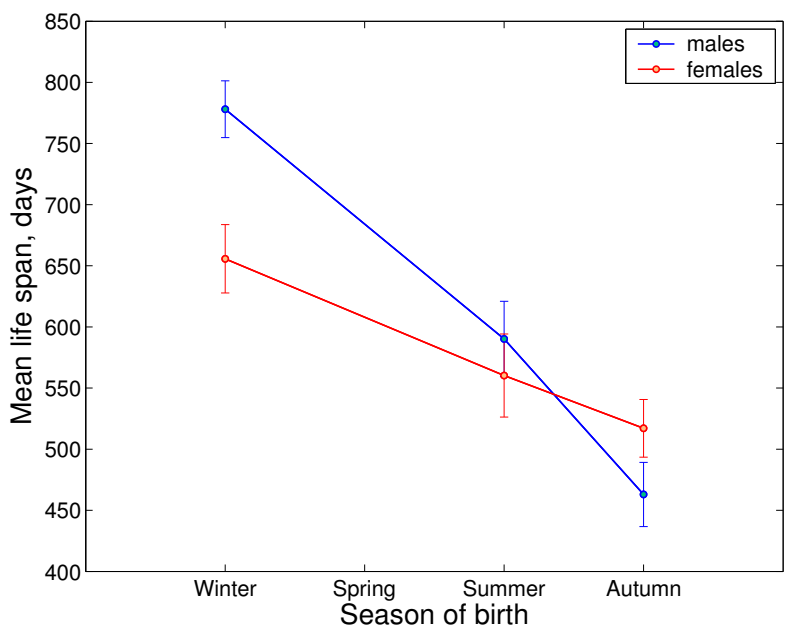

a. all rats

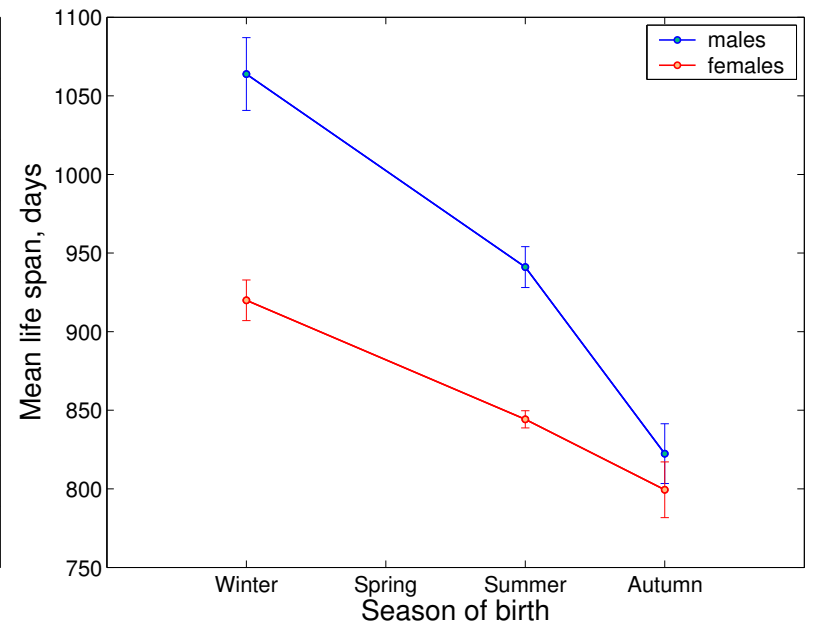

b. last $25 \%$

Figure 6: Mean life span of rats by season of birth

According to the log-rank test, the difference in survival distributions among all groups is statistically significant ( $p=1.19 e-11$ for males and $p=1.60 e-6$ for females). The pairwise difference is also significant with p-values $p=1.29 e-4, p=5.56 e-12$ and $p=1.61 e-3$ for winter-summer, winter-autumn and summer-autumn groups of males, respectively, and $p=3.35 e-3, p=3.69 e-6$ and $p=0.0519$ for females of the same seasons of birth. According to the Cox's proportional hazard model, being born in summer almost doubles the risk of death 
$\left(e^{\beta}=1.83, p=1.4 e-4\right)$, and being born in autumn almost triples it $\left(e^{\beta}=2.78, p=3.2 e-11\right)$ for male rats. The values of relative risk of death for the summer and autumn-born females are not so high, but also significant $\left(e^{\beta}=1.68, p=2.6 e-3, e^{\beta}=1.93, p=5.6 e-6\right)$

Probabilities of death, smoothed with a moving average, for males and females born in different seasons are presented in Figure 7. It can be seen that the probabilities of dying for

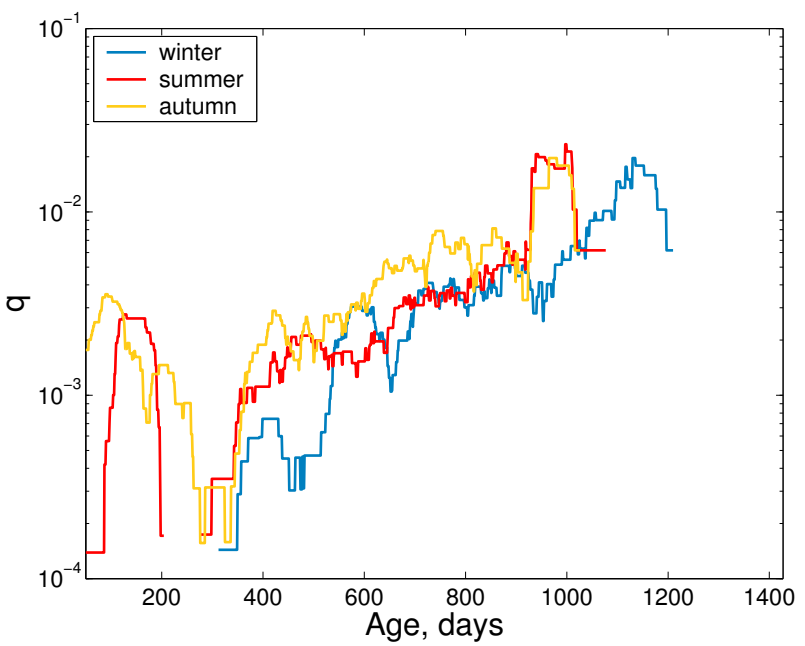

a. males

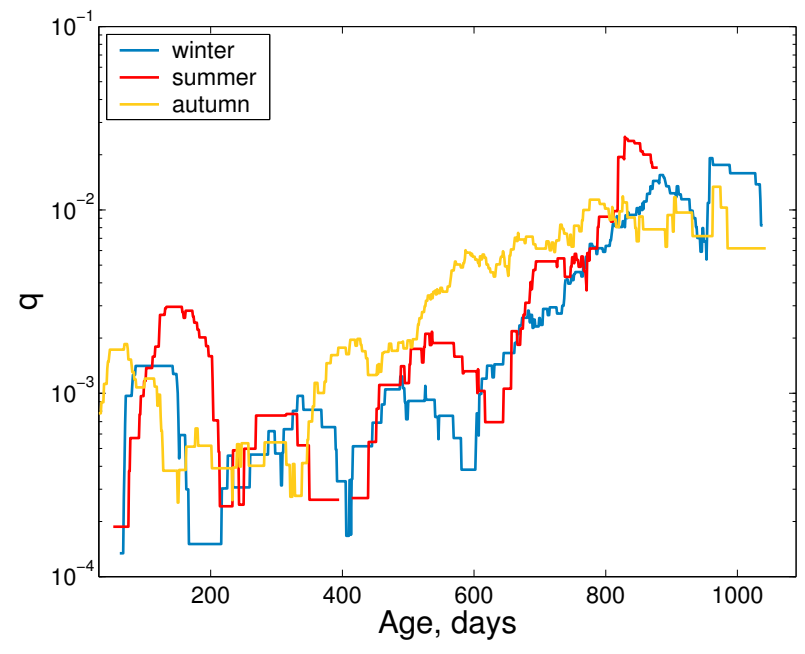

b. females

Figure 7: Probabilities of death (log scale) for rats born in different seasons

the animals born in autumn are higher at almost all ages than for those born in other seasons. It should be noticed, that male rats born in winter started to die at the age of about 350 days, while in the groups born in summer and autumn there are peaks of mortality at young ages. In the groups of male rats born in summer and autumn the probabilities of death reached such values again at the age of about 650 days. Among females, the peaks of mortality are observed in all seasons, but probability of death in the winter-born group at its maximum for young ages is lower than in the other groups. The probabilities of death reached the same levels again at the age of about 420, 660 and 680 days in the autumn, winter and summer groups of birth, respectively. The periods of increased mortality at young ages are followed by the periods, during which no deaths were observed. We assume, that those peaks of mortality at young ages are due to stresses experienced by animals in laboratories, because, even serving as a control, they are subjected to various manipulations such as palpation, weighing, taking smears etc., so weak individuals simply die out.

The hypothesis about stratification of the populations of rats into weak and robust subgroups was tested using the discrete heterogeneity model. Estimated parameters of this model are given in Table 6 . The modeling results confirmed that such a stratification exists in all season-of-birth groups of rats, except the group of winter-born males, in which weak subgroup could not be marked out. The proportion of weak individuals among male rats increases from summer to autumn. In female rats, the proportion of weak individuals in the winter-born group is the lowest, in the summer-born group - the highest, and in the autumn-born group is between two others.

Weak subgroup of male rats born in summer has lower initial risk of dying $\left(\alpha_{w}\right)$, but it increases with age much more rapidly $\left(\beta_{w}\right)$ than in the autumn-born subgroup. Initial values of mortality $\left(\alpha_{r}\right)$ for robust males are equal for the winter and summer-born groups, and they are lower than initial mortality in the group born in autumn. The rate of mortality increase with age $\left(\beta_{r}\right)$ is greater for the summer-born animals, and equal in two other groups.

Among female rats, the initial mortality in weak subgroups $\left(\alpha_{w}\right)$ increases from winter 
to autumn, and the rate of its increase with age $\left(\beta_{w}\right)$ is greater in the summer-born group. Robust females born in summer have the lowest initial mortality $\left(\alpha_{r}\right)$, which increases with age faster $\left(\beta_{r}\right)$ than in two other groups. Initial mortality in the autumn-born subgroup $\left(\alpha_{r}\right)$ is the greatest among all robust female subpopulations. The slowest rate of mortality increase with age $\left(\beta_{r}\right)$ is inherent to the winter-born robust female subpopulation.

Using estimated values of $p_{w}$, the weak subpopulations were removed from each season-of birth groups of rats. The semi-parametric model of heterogeneous mortality were applied for comparison of robust subgroups, given males and females survived the age of 350 days and 250 days, respectively.

Table 6: Estimated parameters of the discrete heterogeneity model for populations of rats born in different seasons

\begin{tabular}{lllllll}
\hline \multicolumn{5}{c}{ males } & & females \\
& winter & summer & autumn & winter & summer & autumn \\
\hline$\alpha_{w}$ & - & $6 \mathrm{e}-7( \pm 6 e-8)$ & $5 \mathrm{e}-3( \pm 1 e-4)$ & $2 \mathrm{e}-6( \pm 4 e-7)$ & $8 \mathrm{e}-5( \pm 5 e-6)$ & $2 \mathrm{e}-2( \pm 3 e-3)$ \\
$\beta_{w}$ & - & $1 \mathrm{e}-1( \pm 2 e-2)$ & $4 \mathrm{e}-2( \pm 5 e-3)$ & $1 \mathrm{e}-1( \pm 3 e-2)$ & $5 \mathrm{e}-2( \pm 2 e-3)$ & $1 \mathrm{e}-2( \pm 2 e-3)$ \\
$\alpha_{r}$ & $1 \mathrm{e}-4( \pm 3 e-5)$ & $1 \mathrm{e}-4( \pm 4 e-5)$ & $4 \mathrm{e}-4( \pm 5 e-4)$ & $4 \mathrm{e}-5( \pm 8 e-6)$ & $2 \mathrm{e}-5( \pm 6 e-6)$ & $2 \mathrm{e}-4( \pm 7 e-5)$ \\
$\beta_{r}$ & $4 \mathrm{e}-3( \pm 2 e-4)$ & $5 \mathrm{e}-3( \pm 3 e-4)$ & $4 \mathrm{e}-3( \pm 2 e-3)$ & $6 \mathrm{e}-3( \pm 3 e-4)$ & $8 \mathrm{e}-3( \pm 5 e-4)$ & $5 \mathrm{e}-3( \pm 5 e-4)$ \\
$p_{w}$ & 0 & $0.18( \pm 0.01)$ & $0.23( \pm 0.01)$ & $0.11( \pm 0.01)$ & $0.24( \pm 0.01)$ & $0.15( \pm 0.01)$ \\
\hline
\end{tabular}

According to the likelihood ratio statistics, the specification of the semi-parametric model of heterogeneous mortality, which takes into account differences in the baseline hazard and both parameters of the frailty distribution, fits the data better than two others (respective $p$-values for the models $r, \sigma^{2}$ and $\alpha, r, \sigma^{2}$ are 0.0012 and 0.0036$)$. Estimated values of the semi-parametric heterogeneous mortality model are given in the Table 7 . Fit of the model to empirical survival functions is presented in Figure 8.

Table 7: Estimated parameters of the heterogeneous mortality model for robust subgroups of rats born in different seasons

\begin{tabular}{|c|c|c|}
\hline & \multicolumn{2}{|c|}{ season of birth } \\
\hline & summer & autumn \\
\hline \multicolumn{3}{|c|}{ male rats } \\
\hline$\alpha$ & $0.1798(0.1781 ; 0.1803)$ & $0.076(0.074 ; 0.077)$ \\
\hline$\beta$ & $0.0043(0.004 ; 0.0045)$ & $0.0037(0.0034 ; 0.0038)$ \\
\hline$r$ & $0.00429(0.00428 ; 0.00432)$ & $0.0179(0.0177 ; 0.0184)$ \\
\hline$\gamma$ & $0.00678(0.00672 ; 0.00684)$ & $0.0498(0.04980 .0498 ;)$ \\
\hline$\sigma^{2}$ & \multicolumn{2}{|c|}{$0.2495(0.2462 ; 0.2507)$ for all seasons } \\
\hline \multicolumn{3}{|c|}{ female rats } \\
\hline$\alpha$ & $0.00156(0.00155 ; 0.00159)$ & $0.0075(0.0071 ; 0.0078)$ \\
\hline$\beta$ & $0.037(0.0363 ; 0.0375)$ & $0.0086(0.0083 ; 0.0089)$ \\
\hline$r$ & $1.139(1.135 ; 1.141)$ & $0.047(0.046 ; 0.049)$ \\
\hline$\gamma$ & $0.897(0.892 ; 0.899)$ & $2.447(2.445 ; 2.448)$ \\
\hline$\sigma^{2}$ & \multicolumn{2}{|c|}{$0.2732(0.2728 ; 0.2737)$ for all seasons } \\
\hline
\end{tabular}

It can be seen that the female population born in winter is more heterogeneous than the male one $\left(\sigma^{2}\right)$. Both male and female rats born in summer or autumn have increased baseline hazard $(\alpha>0$ and $\beta>0)$ compared to respective winter-born groups. Summer and autumnborn males are more robust on average $(r<1)$ and both populations are more homogeneous 
$(\gamma<1)$ than the winter-born group. As for females, the summer-born group is frailer on average $(r>1)$ and less heterogeneous $(\gamma<1)$ than the winter-born population. The autumn-born females are more robust on average $(r<1)$, and this population is much more heterogeneous $(\gamma>1)$ than the one born in winter.

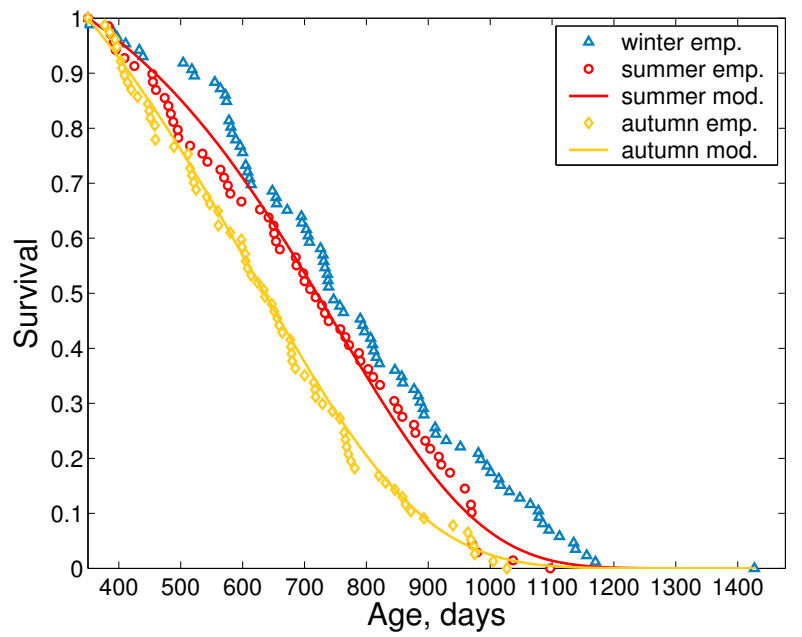

a. males

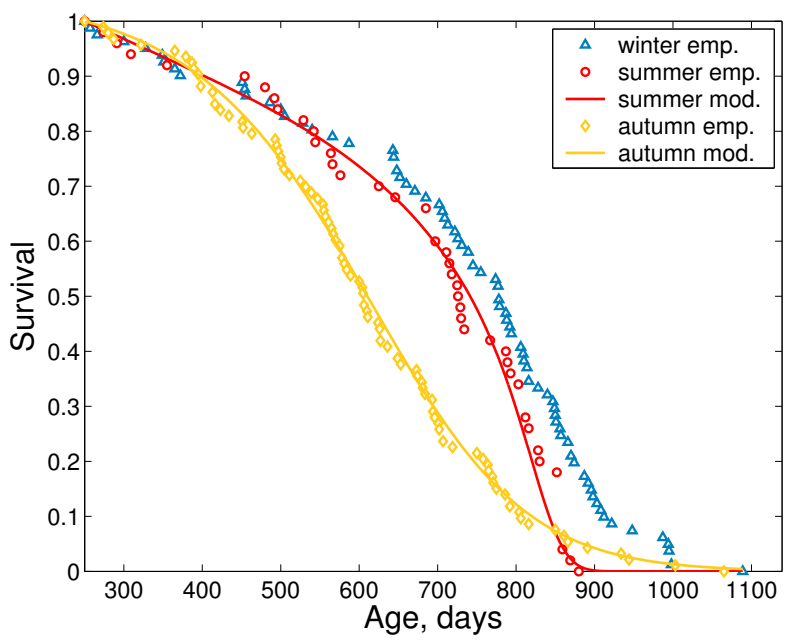

b. females

Figure 8: Empirical and modeled survival

The baseline hazard ( $\alpha>0$ and $\beta>0$ ) is greater in the summer-born group of male rats, however these animals are more robust on average $(r<1)$ than the autumn-born ones. The robust subpopulation of summer-born male rats is more homogeneous $(\gamma<1)$ than subgroup born in autumn. This explains why only a few animals from the summer-born group lived longer than rats born in autumn. Among robust subgroups of female rats the increase of the baseline hazard $(\alpha>0)$ at young ages is greater in the autumn-born population, but its amplification with age $(\beta>0)$ is greater in the group born in summer. Due to decreased heterogeneity $(\gamma<1)$ the smallest maximal life span was observed in the summer-born group. Increased heterogeneity $(\gamma>1)$ of the autumn-born population ensures the presence of the individuals, whose life span is almost as long as in the winter-born group.

\subsection{Summary}

In our study being born in winter is most favorable for the survival of laboratory animals. There is one exception, namely male flies: they experience slightly higher survival when they are born in autumn. The differences in sex-mortality response of medflies subjected to dietary manipulations were studied before (Carey, 2003; Carey et al., 2001), and they may account for different patterns in changes of the baseline hazard and frailty distribution with season in male population, compared to the female one. The worst seasons for medflies are spring and summer, for female mice - summer and autumn, and for rats - summer.

The analysis of the baseline hazard, average frailty and population heterogeneity reveals two common features (Table 8). First, among all species those born in seasons less favorable for survival experience an increased baseline hazard and an amplification of mortality with age. Second, they are usually more robust on average, which may reflect the ability of the individual to adapt to less favorable environmental conditions. Among flies these two features coincide with larger population heterogeneity. Among rodents there is a tendency towards lower heterogeneity, however, the most heterogeneous female populations were born in the worst season (summer for mice, autumn for rats). 
Table 8: Summary of the Parameter Estimates of the semi-parametric survival model

\begin{tabular}{|c|c|c|c|c|}
\hline Season & Hazard function & Amplification with age & Frailty & Heterogeneity \\
\hline \multicolumn{5}{|c|}{ Male Flies (maximum LE: winter/autumn; minimum LE: spring/summer) } \\
\hline Spring & - & - & + & + \\
\hline Summer & + & + & - & ++ \\
\hline Autumn & - & - & + & + \\
\hline \multicolumn{5}{|c|}{ Female Flies (maximum LE: winter/autumn; minimum LE: spring/summer) } \\
\hline Spring & + & + & - & + \\
\hline Summer & + & + & - & + \\
\hline Autumn & + & + & -- & ++ \\
\hline \multicolumn{5}{|c|}{ Female Mice (maximum LE: winter/spring; minimum LE summer/autumn) } \\
\hline Spring & + & + & -- & - \\
\hline Summer & + & + & - & ++ \\
\hline Autumn & ++ & + & - & -- \\
\hline \multicolumn{5}{|c|}{ Male Rats (maximum LE: winter; minimum LE summer/autumn) } \\
\hline Summer & ++ & + & -- & -- \\
\hline Autumn & + & + & - & - \\
\hline \multicolumn{5}{|c|}{ Female Rats (maximum LE: winter; minimum LE summer/autumn) } \\
\hline Summer & + & ++ & + & - \\
\hline Autumn & ++ & + & - & ++ \\
\hline
\end{tabular}

\section{Discussion}

Among the three species - flies, rats and mice — we found a significant pattern in changes in mean life span by month of birth or month of eclosion. This pattern is unexpected insofar, as that all observed animals belong to the control groups of experiments that tried to control for confounding environmental factors. The observed seasonal patterns share several characteristics in terms of life expectancy, baseline hazard, average frailty and population heterogeneity. This similarity exists despite the fact that climate conditions differ largely between the laboratories in St. Petersburg, where the experiments on mice and rats were conducted, and Chiapas, where the flies were reared.

We analyzed life span by season of birth/eclosion rather than by month or week of birth/eclosion. One reason is that we do not have enough observations for a more detailed seasonal analysis of mice and rats. A second reason is that the data on rats and mice only include the month of birth rather than the exact date of birth. A third reason is that this categorization allows us to assume that gestation period falls in the same season as birth/eclosion. Therefore season of birth measures both factors that fall into the fetal development as well as in the very first period after birth. The drawback is that we cannot distinguish which one of the two periods is more important.

\subsection{Month-of-birth patterns in humans}

The pattern that we observe among flies, rats and mice in this study reflects the pattern in life span by month of birth found in earlier studies on human populations. As an example we show the birth pattern in the remaining life expectancy at the age of 50 of the Danish population (Figure 4.1). This pattern is based on Danish register data with a mortality follow-up of all 
Danes who were at least 50 years old on 1 April 1968. This is a total of 1,371,003 people, who were followed up to week 32 of 1998.

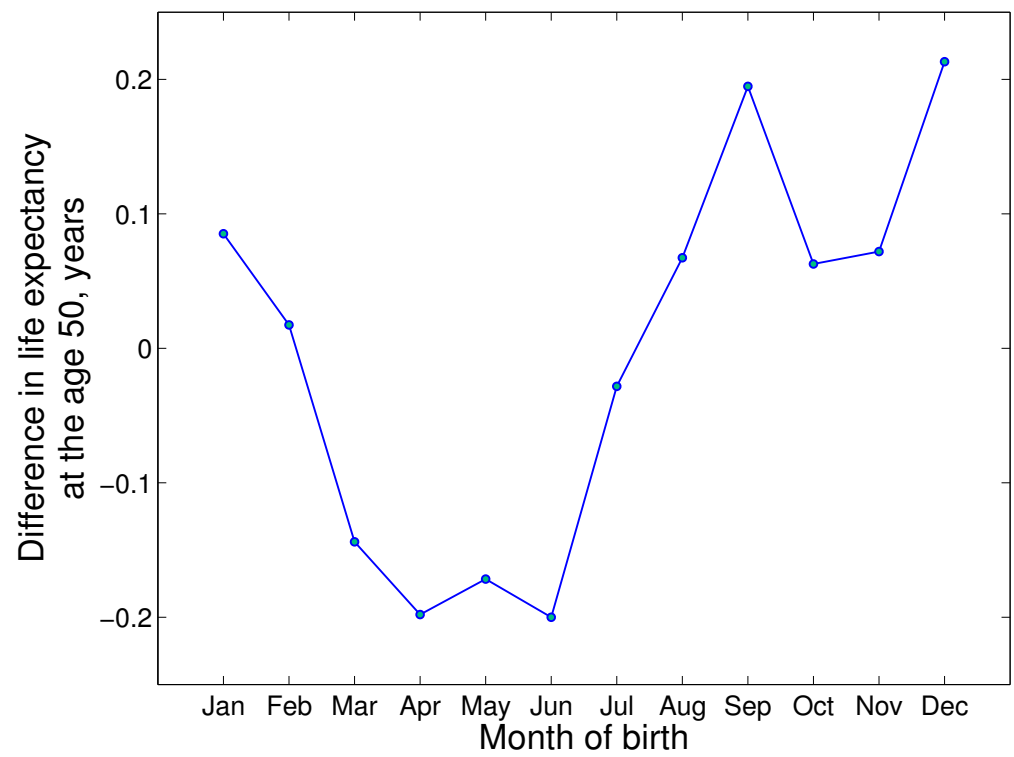

Figure 9: Deviation of remaining life expectancy at age 50 for people born in a specific month from average remaining life expectancy for Denmark 1968 -1998.

A similar pattern exists for the US population and based on about 15 Mio. death certificates (Doblhammer, 2004). The US death certificates also include information about educational status of the deceased and it was therefore possible to distinguish the month-of-birth pattern by educational group. There is a clear tendency that the pattern decreases with increasing education.

Earlier Gavrilov and Gavrilova (1999) reported that month of birth is an important predictor of life expectancy of adult women (30 years and above), in particular, women born in May and December tend to live 3 years longer on average compared to those born in August. These findings are based on the analysis of 4,911 genealogical longevity records for women born in 1800-1880 from European aristocratic families (as described in Gavrilova et al., 1998).

Later Vaiserman et al. (2002) studied the association between longevity and season of birth in 101,634 individuals who died in Kiev (Ukraine) during the period 1990-2000. The relationship between age at death and month of birth showed a very similar pattern for both men and women. Mean values for the age at death were lowest for individuals born in AprilJuly, and highest for those born at the beginning and end of the year. Minimal and maximal ages at death, analyzed according to month of birth, differed by 2.6 years in men and 2.3 years in women. For all major causes of death, the mean age at death for persons born in winter was the highest.

\subsection{Causal factors}

The season-of-birth dependent pattern of longevity reported by Gavrilov and Gavrilova (1999) differs form the one found by Doblhammer and Vaupel (2001) and Vaiserman et al. (2002) probably due to the specificity of the studied population - aristocrats born in $19^{\text {th }}$ century. Gavrilov and Gavrilova (1999) explained their finding by the reliability theory of aging, which emphasize the importance of initial level of damage in determining future length of human life (Gavrilov and Gavrilova, 1991). 
Vaiserman et al. (2002) also suggested that in studied population longevity was affected by prenatal or early postnatal seasonal factors. Authors noted that their finding is consistent with the hypothesis that the rate of ageing may be programmed in response to environmental influences at critical periods of early development.

Previous research (Doblhammer, 2004; Doblhammer and Vaupel, 2001) has shown that the month-of-birth pattern among the adult Danish population today is positively correlated with the month-of-birth pattern in survival during the first year of life of the respective cohorts born at the beginning of the $20^{\text {th }}$ century. This finding suggests that debilitating factors at the beginning of life are responsible for the month-of-birth pattern in survival at adult ages: infants born in spring had a higher risk to die during their first year of life than those born in other seasons. Those who survived were debilitated and had higher mortality risks later in life. Seasonal differences in the diet of the pregnant mother and the seasonal incidence in infectious disease have been suggested as the underlying factors.

It is unclear, however, whether these factors are also responsible for the month-of-birth patterns in the life spans of laboratory flies, rats and mice. For laboratory animals diet and environmental conditions are strictly controlled. They are fed standardized diet throughout the year. However, taking into account that fruit flies are very sensitive to diet (Carey, 2003; Carey et al., 2002; Romanyukha et al., 2004), we cannot exclude the possibility that micronutrients vary with the seasons. As for mice, season variation in food qualilty also possibly can take a place, but in our experiments animals were given standard laboratory chow and was not supplemented with "season food" (vegetables, fruits, grass, green leaves, etc.) Rats were fed a diet cooked of natural grain, meat and milk ("kasha") and vegetables. The quality of this diet could possipbly vary with season. Infection diseases are not common in vivariums - if yes animals died rapidly.

Other mechanisms are possible too. It has been shown that the seasonal changes in the hours of daylight influence the human neuroendocrine functions, in particular melatonin production (Wehr, 1997, 1998). Because daylength changes throughout the year there are seasonal effects on the melatonin rhythm in experimental animals and men, irrespective of whether the animal being studied is nocturnally or diurnally active (Reiter, 1986). Large seasonal variations in the levels and daily rhythm amplitudes of the different indole metabolites were found in the pineal glands of Djungarian hamsters (Steinlechner et al., 1995). The highest levels of retinal melatonin were found in June (the lowest in December) as opposed to the highest levels of pineal activity in November-February and lowest in April-August (Reiter, 1978).

Prenatal influence of the photoperiod on the ontogeny of melatonin in humans was suggested by Sivan et al. (2001), who found a significant effect of the month of birth on the nocturnal urinary excretion of the major melatonin metabolite, 6 -sulfatoxymelatonin (6SMT) in healthy infants at the age of $8 \mathrm{wk}$. Seasonal changes of nocturnal 6-sulfatoxymelatonin (aMT6s) excretion with peak levels in summer were also found in female rats (Bartsch et al., 1994). It is also of note that these findings were made in laboratory animals despite constant environmental conditions and constant photoperiods (Bartsch et al., 1994).

It is known that urinary excretion of 6-sulfatoxymelatonin can reflect the production of melatonin mainly by enterocharomaffine cells in gastrointestinal tract but not pineal production (Bubenik, 2001). Although research on gastrointestinal melatonin is still inconclusive, periodicity of gastrointestinal melatonin may be more directly related to food intake rather than light exposure. Higher peripheral and tissue levels of melatonin were observed not only after food intake but also after a long-term food deprivation. It is also possible that high level of gastrointestinal melatonin can reflect the low level of pineal production (as retinal melatonin does).

It was shown that pubertal development of rats is subject to manipiulation by both light and melatonin. Injections of melatonin given specifically during the period of pubertal de- 
velopment delayed reproductive maturity in all relevant aspects - gonadal growth, hormone levels, pituitary GnRH receptor content, etc. in both male and females (Arendt, 1995, pages 139-141).

Melatonin has recently been shown to possess antioxidative properties (Reiter et al., 2000; Touitou, 2001) and this suggests an idea that increased level of melatonin production in winter is the cause of lengthening life span of animals and insects born/eclosed in winter.

There is a hypothesis that the horizontal component $\mathrm{H}$ of the geomagnetic field may act as a seasonal 'zeitgeber' because $\mathrm{H}$ shows a similar seasonal rhythm to pineal melatonin production, and changes in the direction and intensity of $\mathrm{H}$ can affect pineal activity (Bartsch et al., 1994).

Cornlissen et al. (2003) reported the similarity of cycle lengths of geomagnetic activity with strong half-yearly component in its spectrum and a circasemiannual pattern in status epilepticus, in several morbid oral conditions, in the cell density of vasopressin-containing neurons in the human suprachiasmatic nuclei (SCN), in circulating melatonin, and in an unusual circasemiannual aspect of a birth-month-dependence of human longevity. But as authors cautiously pointed out, the similarity of cycle lengths in itself can only be a hint prompting the search for causal relations.

Several researchers have postulated that the widely observed winter-spring birth excess in schizophrenia and bi-polar disorders might be caused by variations in internal chemistry or neural development brought about by seasonal variations in light.

Kay (2004) hypothesized that seasonal variation in the geomagnetic field of the earth primarily as a result of geomagnetic storms at crucial periods in intrauterine brain development could affect the later rate of development of schizophrenia.

\subsection{Gene-environment interaction}

Genetic and environmental factors, as well as their interactions, are likely to be involved in psychiatric disorders. Considerable progress has been made in association and linkage studies with various candidate genes, at times with conflicting or ambiguous results. An environmental factor that has persistently shown associations with several psychiatric and neurological disorders is the season of birth. The results of Chotai et al. (2003) suggest an interaction between the seasons of birth and the expression of the candidate genes, and that season of birth is a confounding variable when investigating the role of the candidate genes in susceptibility to psychiatric disorders.

Seeger et al. (2004) found an interaction between the seasons of birth and the expression of the dopamine D4 receptor DRD4 candidate gene in children with hyperkinetic disorder and conduct disorder (HD + CD) as well as in controls, which differ significantly from each other. Depending on the season of birth, children carrying the seven-repeat long variant of this gene $(\mathrm{DRD} 4 * 7 \mathrm{R})$ allele showed different relative risks for developing HD + CD.

Franzek and Beckmann (1996) studied the gene-environment interaction in schizophrenia and found that season-of-birth effect reveals etiologically different subgroups: on the one hand, in sporadic forms of the disease exogenous noxious agents may be of major etiological importance; on the other hand, in fetuses at high genetic risk neurodevelopment may already be disturbed due to a genetic defect and additional environmentally noxious agents can cause abortions, stillbirths and sudden infant deaths.

Another example of gene-environment interaction is the study of 98 Slovak children with type 1 diabetes mellitus (DM1) and their 60 healthy parents and siblings showed that DM1 risk from the insulin gene pattern in births of future diabetics cycled significantly semi-annually, with maxima around the spring and autumn equinoxes (Mikulecky et al., 2004).

Studies on gene-environmental interaction in nonhuman populations should also be mentioned. Seasonal variation (spring, early summer, last summer and autumn) of inversion polymorphisms of the $\mathrm{O}$ chromosome of Drosophila subobscura in a natural population was found 
(Rodriguez-Trelles, 2003; Rodriguez-Trelles et al., 1996; Zapata et al., 2000). Seasonal changes in the frequencies of chromosome arrangements were significantly associated with the seasonal variation of the climate (temperature, rainfall, humidity and insolation) and reflect natural selection.

After advancing the hypothesis that longevity might be programmed by early life exposures (Vaiserman et al., 2002), authors designed an experimental study for the examination of the possibility of longevity programming. Study of adult Drosophila melanogaster DNA repair capacity after irradiation at the egg stage was carried out, using marker such as DNA strand breaks. Insects irradiated in low doses $(0.50$ and $0.75 \mathrm{~Gy})$ had extended life span and increased stability to S1 nuclease treatment (Vaiserman and Voitenko, 2003). Authors offered a possible explanation of observed effects, which implies the long-term modulation of certain (possibly repair) genes activity. They also hypothesize that life-extending effects of different anti-aging treatments might be a consequence of their unspecific (hormetic) action, rather then specific (geroprotector) action on the some aging-related processes, and induction of "transcriptional reprogramming" may be a key mechanism of the longevity programming and artificial life extension.

Though our data on laboratory animals do not allow detailed analysis of gene-environmental interaction, it would be interesting to look at the data on eclosion rate in medflies and number of spontaneous abortions or still births among rodents, depending of season. Nevertheless, it was apparent in the survival of rats, that selection processes and elimination of weak individuals from the population occurred at different rates in considered seasons. Increased population heterogeneity, which was observed in all species in unfavorable for survival seasons, bears the evidence of increased adaptational ability of the species to new environmental conditions on the population level.

\section{Conclusion}

During the last 140 years life expectancy has risen 3 years per decade (Oeppen and Vaupel, 2002). A recent controversial article argues that the reduction in lifetime exposure to infectious disease and other sources of inflammation made an important contribution to the historical decline in old-age mortality (Finch and Crimmins, 2004). The authors show a strong association between early-age mortality and subsequent mortality in the same cohort for cohorts born in Sweden since 1751. The fetal-origins hypothesis of adult disease (Barker et al., 1989) claims that nutrition of the mother during pregnancy or in the first year of life leads to physiological or metabolic programming of the newborn and substantially determines the occurrence of pathological phenomena later in life.

Future trends in mortality improvements among humans hinge critically on the question whether the past improvements where mainly due to improvements in the environment early in life or due to general better living condition during the life course. If the first is the case then it may be argued that future improvements in mortality will be lower than in the past. When measured in terms of infant mortality general living conditions early in life have improved to such a high standard that only minor improvements might be possible in the future. This has important implications on future population developments in particular on population aging.

In addition to the question about the causal mechanisms and the implications for population development there is another important aspect of our findings. If uncontrolled factors such as the month of birth have an important influence on the outcome of laboratory results then this opens general questions about the interpretation of experimental results. 


\section{APPENDIX}

\section{Heterogeneous mortality model}

Let $T$ and $Z$ be the life span and the heterogeneity (frailty) variable such that the conditional hazard of death given $Z$ is $Z \mu_{0}(x)$, where $\mu_{0}(x)$ is the underlying hazard (Vaupel et al., 1979). Let us assume that frailty $Z$ is gamma $(k, \lambda)$ distributed with mean 1 and variance $\sigma^{2}$, i.e. $k=\lambda$, and $\sigma^{2}=\frac{1}{\lambda}$. Let $H(x)=\int_{0}^{x} \mu_{0}(u) d u$ be the cumulative underlying hazard. Then the observed mortality $\bar{h}(x)$ is:

$$
\bar{\mu}(x)=\frac{\mu_{0}(x)}{1+\sigma^{2} H(x)}
$$

The marginal survival function $S(x)$ is:

$$
S(x)=\left(1+\frac{1}{\lambda} H(x)\right)^{-k}=\left(1+\sigma^{2} H(x)\right)^{-\frac{1}{\sigma^{2}}}
$$

In the case of homogeneous population $\sigma^{2}=0$, expression (1) transforms into $\bar{\mu}(x)=\mu_{0}(x)$. Using the L'Hospital's rule, it is easy to show that $S(x) \rightarrow \exp (-H(x))=S_{0}(x)$, when $\sigma^{2} \rightarrow 0$ in (2).

In our further calculations we will follow the methodology for the analysis of data from the stress experiment suggested by Yashin et al. (1996). The application of this model to the analysis of post-stress survival of Drosophila melanogaster flies is described in Semenchenko et al. (2004).

Let us consider two identical heterogeneous populations whose chances of survival correspond to the proportional hazards model and assume that the initial frailties are gammadistributed with means 1 and variances $\sigma_{1}^{2}, \sigma_{2}^{2}$. The first population - the control group experiences standard living conditions without any interventions and the second is subjected to some treatment at the age interval $\left[x_{0}, x^{*}\right]$. To compare the survival functions after age $x^{*}$ in the experimental and in the control group let us assume that in the control group the underlying hazard $\mu_{01}(x)$ does not change and in the experimental cohort the underlying hazard $\mu_{02}(x)$ increases at the interval $\left[x_{0}, x^{*}\right]$ and that after age $x^{*}$ it is $\mu_{02}(x)=\mu_{0}(x)+f(x)$. Note that if $f(x) \equiv 0$ the underlying hazard returns to its standard level, a negative $f(x)$ manifests the presence of adaptive effect, and a positive represents debilitative effects. It follows from (2) that the marginal survival functions $S_{i}(x), i=1,2$ for those who survived age $x^{*}$ are:

$$
S_{i}(x)=\left(1+\frac{1}{\lambda_{i}^{*}} H_{i}^{*}(x)\right)^{-k_{i}}, i=1,2
$$

Where $\lambda_{i}^{*}=\frac{1}{\sigma_{i}^{2}}+H_{i}\left(x^{*}\right), H_{i}\left(x^{*}\right)=\int_{0}^{x^{*}} h_{0 i}(u) d u, H_{1}^{*}(x)=\int_{x^{*}}^{x} h_{0}(u) d u, H_{2}^{*}(x)=\int_{x^{*}}^{x} h_{0}(u) d u+$ $F(x), F(x)=\int_{x^{*}}^{x} f(u) d u$ and $k_{i}=\frac{1}{\sigma_{i}^{2}}, i=1,2$.

Let us assume that under normal living conditions an individual's susceptibility to death does not change during its life and that any exogenous intervention can increase or decrease an individual's frailty.

Note that in the control population even the "natural" selection process does not change the shape parameter $k_{1}=\frac{1}{\sigma_{1}^{2}}$ of the frailty distribution. If the application of treatment does not influence an individual's frailty, this parameter also does not change in the experimental cohort, i.e. $k_{2}=\frac{1}{\sigma_{2}^{2}}$. 
Let us assume that $\sigma_{1}^{2}=\sigma_{2}^{2}=\sigma^{2}$ and that the application of a treatment can also change the shape parameter of the frailty distribution by a factor $\gamma$, i.e. $k_{2}=\frac{1}{\gamma \sigma^{2}}$. Changes in frailty variance reflect non-linear changes in population heterogeneity: this can occur, for example, when weak individuals become weaker, robust individuals increase their robustness, and so on. So, for the survival in the control cohort after age $x^{*}$, one can write:

$$
S_{1}(x)=\left(1+\frac{k_{1}^{*}}{\lambda_{1}^{*}} \sigma^{2} H_{1}^{*}(x)\right)^{-k_{1}^{*}}=\left(1+m_{1}^{*} \sigma^{2} H_{1}^{*}(x)\right)^{-\frac{1}{\sigma^{2}}}
$$

and for the survival in the experimental cohort $\left(x>x^{*}\right)$

$$
S_{2}(x)=\left(1+m_{2}^{*} \gamma \sigma^{2} H_{2}^{*}(x)\right)^{-\frac{1}{\gamma \sigma^{2}}}
$$

where $m_{1}^{*}, m_{2}^{*}$ are the mean values of the frailty distribution in the control and in the experimental populations at age $x^{*}$ respectively, and $\gamma$ is the factor which shows the presence of changes in the frailty distribution that are not associated with changes of average frailty in the population during the treatment.

Note further that it follows from (4) and from the definition of $F(x)$ that

$$
H_{2}^{*}(x)=\frac{S_{1}(x)^{-\sigma^{2}}-1}{\sigma^{2} m_{1}^{*}}+F(x)
$$

Replacing $H_{2}^{*}(x)$ in (5) with (6) we obtain the following equation for the survival $S_{2}(x),\left(x>x^{*}\right)$ in the experimental group:

$$
S_{2}(x)=\left(1+r \gamma\left(S_{1}(x)^{-\sigma^{2}}-1\right)+m_{1}^{*} r \gamma \sigma^{2} F(x)\right)^{-\frac{1}{\gamma \sigma^{2}}}
$$

with $r=\frac{m_{2}^{*}}{m_{1}^{*}}$. From the definition of $\mu(x)=-S^{\prime}(x) / S(x)$ and expression (7) we can derive the representation of observed mortality in experimental group:

$$
\bar{\mu}_{2}(x)=\frac{r \bar{\mu}_{1}(x) S_{1}(x)^{-\sigma^{2}}+m_{1}^{*} r f(x)}{1+r \gamma\left(S_{1}(x)^{-\sigma^{2}}-1\right)+m_{1}^{*} r \gamma \sigma^{2} F(x)}
$$

In our calculations we use $f(x)=a e^{\beta\left(x-x^{*}\right)}$. Denoting $\alpha=a m_{1}^{*}$, equations (7) and (8) can be rewritten as:

$$
\begin{gathered}
S_{2}(x)=\left(1+r \gamma\left(S_{1}(x)^{-\sigma^{2}}-1\right)+\gamma r \sigma^{2} \frac{\alpha}{\beta}\left(e^{\beta\left(x-x^{*}\right)}-1\right)\right)^{-\frac{1}{\gamma \sigma^{2}}} \\
\bar{\mu}_{2}(x)=\frac{r \bar{\mu}_{1}(x) S_{1}(x)^{-\sigma^{2}}+r \alpha e^{\beta\left(x-x^{*}\right)}}{1+r \gamma\left(S_{1}(x)^{-\sigma^{2}}-1\right)+\gamma r \sigma^{2} \frac{\alpha}{\beta}\left(e^{\beta\left(x-x^{*}\right)}-1\right)}
\end{gathered}
$$

Parameter $\sigma^{2}$ indicates the presence of heterogeneity in the control population. Different baseline survival patterns which can resume in the same survival function for the control group, depending on heterogeneity of the latter, are presented in Figure 10.a. If $\sigma^{2} \rightarrow 0$, the control group becomes homogeneous, and $S_{c} \rightarrow S_{0}$ (See the Appendix). With an increase of the frailty variance the survival function for the control group shifts to the right along the age axis with a noticeable increase of the tail.

Effects of changes in the baseline hazard, controlled by parameters $\alpha$ and $\beta$, are presented in Figures $10 . \mathrm{b}-\mathrm{d}$. If $\beta=0$ in the additive part of hazard for the treatment group $f(x)=$ $a \exp (\beta x)$, changes in parameter $\alpha$ reflect permanent (constant) decrease or increase of the 

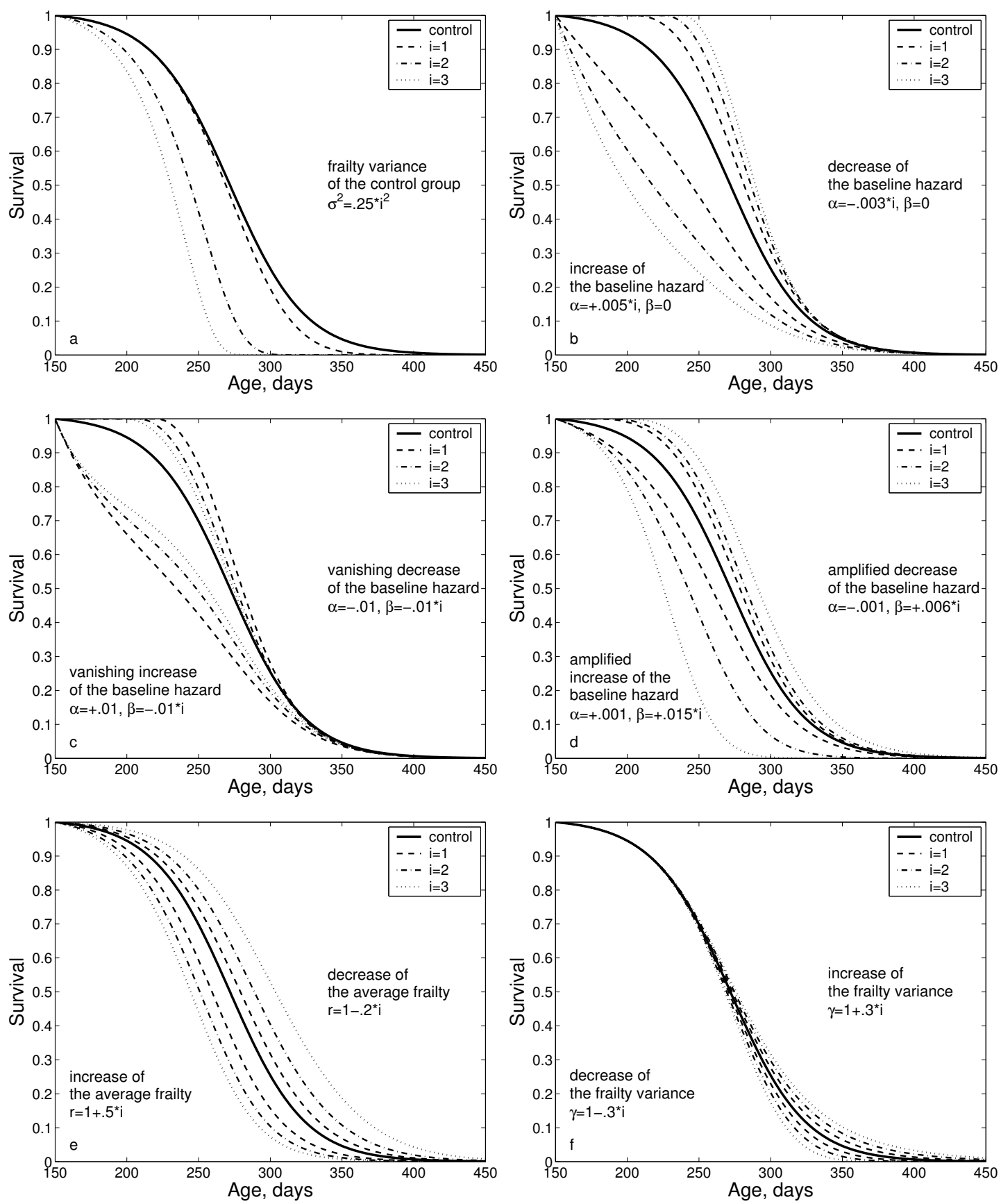

Figure 10: Different baseline (a) and treatment groups survival patterns (b-f) depending on changes of the model's parameters. 
baseline hazard, producing rectangularization or derectangularization of the survival curve, respectively, depending on whether $\alpha$ is greater or less than zero (Fig. 10.b). In our study we call these effects debilitation or adaptation, depending on increase or decrease of baseline hazard. It can be seen in Figure 10.b that constant debilitation and adaptation do not influence the 'tail' of the survival curve.

Parameter $\beta$ describes the amplification or disappearance of the $\alpha$-effect, according to whether $\beta$ is greater or less than zero. For each effect small value of $\alpha$ was fixed. Vanishing debilitative and adaptive effects are shown in Figure 10.c. A decrease of negative $\beta$ draws the survival curve for the treatment group closer to the survival curve for the control group. Vanishing effects of debilitation and adaptation also do not shift the tail of survival function. Amplified debilitation and adaptation are shown in Figure 10.d. An increase of positive $\beta$ shifts the survival curve to the left along the age axis (compared to the control group) in case of amplified debilitation, and to the right in case of amplified adaptation. In both cases the tail of survival curve moves in the same direction. The shifts produced are not parallel, they resemble rotation around the initial level of debilitation or adaptation.

Effects of changes in the frailty distribution are presented in Figures 10.e-f. An increase or decrease in mean of the frailty distribution produces nearly parallel shift of the survival curve along the age axis with respective lengthening/shortening of its tail (Fig. 10.e). Parameter $r<1$ shows an increase in the average robustness, while $r>1$ indicates an accumulation of frail individuals in the population. Parameter $\gamma \neq 1$ shows an increase $(\gamma>1)$ or decrease $(\gamma<1)$ in the population heterogeneity. These effects influence mostly the tail of survival function (Fig. 10.f).

\section{References}

Anisimov, V., G. Pliss, M. Iogannsen, I. Popovich, K. Romanov, and T. Averyanova (1989). Spontaneous tumors in outbred lio rats. J.Exp.Clin. Cancer Res. 8(4), 254-262.

Anisimov, V. N., I. N. Alimova, D. A. Baturin, I. G. Popovich, M. A. Zabezhinski, K. G. Manton, A. V. Semenchenko, and A. I. Yashin (2003). The effect of melatonin treatment regimen on mammary adenocarcinoma development in HER-2/neu transgenic mice. International Journal of Cancer 103, 300-305.

Arendt, J. (1995). Melatonin and the Mammalian Pineal Gland. London: Chapman \& Hall.

Baranova, L., K. Romanov, and V. Jamshanov (1985). Study of levels of benzo(a)pyrene and n-ntrosamines in the food of laboratory animals. Vopr. Onkol. 5, 54-57.

Barker, D., C. Osmond, J. Golding, D. Kuh, and W. M.E.J. (1989). Growth in-utero, blood pressure in childhood and adult life, and mortality from cardiovascular disease. British Medical Journal 298(6673), 564-567.

Bartsch, H., C. Bartsch, D. Mecke, and T. Lippert (1994, Feb). Seasonality of pineal melatonin production in the rat: possible synchronization by the geomagnetic field. Chronobiol Int. 11(1), 21-26.

Bubenik, G. A. (2001). Localization, physiological significance and possible clinical implication of gastrointestinal melatonin. Neurosignals 10(6).

Carey, J. R. (2003, February). Longevity: The Biology and Demography of Life Span. Princeton University Press.

Carey, J. R., P. Liedo, L. Harshman, Y. Zhang, H.-G. Müller, L. Partridge, and J. L. Wang (2002). Life history response of mediterranean fruit flies to dietary restriction. Aging Cell. 
Carey, J. R., P. Liedo, H.-G. Müller, J.-L. Wang, B. Love, L. Harshman, and L. Partridge (2001). Female sensitivity to diet and irradiation treatments underlies sex-mortality differentials in the mediterranean fruit fly. Journal of Gerontology: Biological Sciences 56A(2), B89-B93.

Chotai, J., A. Serretti, E. Lattuada, C. Lorenzi, and R. Lilli (2003). Gene-environment interaction in psychiatric disorders as indicated by season of birth variations in tryptophan hydroxylase (TPH), serotonin transporter (5-HTTLPR) and dopamine receptor (DRD4) gene polymorphisms. Psychiatry Research 119, 99-111.

Cornlissen, G., F. Halberg, L. Pllmann, B. Pllmann, G. S. Katinas, H. Minne, T. Breus, R. B. Sothern, Y. Watanabe, R. Tarquini, F. Perfetto, C. Maggioni, D. Wilson, D. Gubin, K. Otsuka, and E. E. Bakken (2003). Circasemiannual chronomics: half-yearly biospheric changes in their own right and as a circannual waveform. Biomedicine $\&$ Pharmacotherapy 5\%, $45 \mathrm{~s}-54 \mathrm{~s}$.

Cox, D. (1972). Regression models and life-tables (with discussion). Journal of the Royal Statistical Society. Series B (Methodological) 34(2), 187-220.

Cox, D. and D. Oakes (1988). Analysis of survival data. Chapman and Hall. London.

Davison, A. C. and D. V. Hinkley (1997). Bootstrap Methods and Their Application. Cambridge, England: Cambridge University Press.

Doblhammer, G. (2004). The Late Life Legacy of Very Early Life. Heidelberg: Springer Verlag.

Doblhammer, G. and J. Vaupel (2001). Lifespan depends on month of birth. PNAS 98(5), 2934-2939.

Finch, C. and E. Crimmins (2004). Inflammatory exposure and historical changes in human life-spans. Science 305, 1736-1739.

Fletcher, R. (1987). Practical methods of optimization. John Wiley \& Sonh, New York, second edition.

Franzek, E. and H. Beckmann (1996). Gene-environment interaction in schizophrenia: seasonof-birth effect reveals etiologically different subgroups. Psychopathology 29(1), 14-26.

Gavrilov, L. A. and N. Gavrilova (1991). The Biology of Life Span: a Quantitative Approach. New York: Harwood Academic Publisher.

Gavrilov, L. A. and N. Gavrilova (1999). Season of birth and human longevity. Journal of anti-aging medicine 2(4), 365-366.

Gavrilova, N., L. Gavrilov, G. Evdokushkina, et al. (1998). Evolution, mutations and human longevity: European royal and noble families. Hum Biol 70, 799-804.

Gompertz, B. (1825). On the nature of the function expressive of the law of human mortality, and on the new mode of determining the values of life contingencies. Philosophical Transaction, The Royal Society, London 115, 513-585.

Kalbfleisch, J. D. and R. L. Prentice (1980). The Statistical Analysis of Failure Time Data. John Wiley \& Sons, Inc, New York.

Kay, R. W. (2004). Schizophrenia and season of birth: relationship to geomagnetic storms. Schizophrenia Research 66, 7-20. 
Mikulecky, M., P. Minarik, and D. Michalkova (2004, May). Insulin gene profile cycles with season of birth of future diabetic children and their relatives. J Pediatr Endocrinol Metab 17(5), $727-730$.

Oeppen, J. and J. W. Vaupel (2002, 10 May). Broken limits to life expectancy. Science 296, 1029-1031.

Reiter, R. (1978). Interaction of photoperiod, pineal gland and seasonal reproduction as exemplified by finding in the hamster. Prog Reprod Biol 4, 169-190.

Reiter, R. (1986). Normal patterns of melatonin levels in the pineal gland and body fluids of humans and experimental animals. J Neural Transm Suppl 21, 35-54.

Reiter, R., D.-X. Tan, C. Osuna, and E. Gitto (2000). Actions of melatonin in the reduction of oxidative stress. J Biomed Sci 7, 444-458.

Rodriguez-Trelles, F. (2003, Apr). Seasonal cycles of allozyme-by-chromosomal-inversion gametic disequilibrium in Drosophila subobscura. Evolution Int J Org Evolution 57(4), 839848.

Rodriguez-Trelles, F., G. Alvarez, and C. Zapata (1996, Jan). Time-series analysis of seasonal changes of the o inversion polymorphism of Drosophila subobscura. Genetics 142(1), 179187.

Romanyukha, A., J. Carey, A. Karkach, and A. Yashin (2004). The impact of diet switching on resource allocation to reproduction and longevity in mediterranean fruitflies. Proc. $R$. Soc. Lond. B 271, 1319-1324.

Seeger, G., P. Schloss, M. H. Schmidt, A. Rter-Jungfleisch, and F. A. Hennd (2004). Geneenvironment interaction in hyperkinetic conduct disorder $(\mathrm{HD}+\mathrm{CD})$ as indicated by season of birth variations in dopamine receptor (DRD4) gene polymorphism. Neuroscience Letters $366,282-286$.

Semenchenko, G. V., A. A. Khazaeli, J. W. Curtsinger, and A. I. Yashin (2004). Stress resistance declines with age: Analysis of data from survival experiment with Drosophila melanogaster. Biogerontology 5(1), 17-30.

Sivan, Y., M. Laudon, R. Tauman, and N. Zisapel (2001, Jan). Melatonin production in healthy infants: evidence for seasonal variations. Pediatr Res. 49(1), 63-68.

Steinlechner, S., I. Baumgartner, G. Klante, and R. Reiter (1995, Sep). Melatonin synthesis in the retina and pineal gland of Djungarian hamsters at different times of the year. Neurochem Int 27(3), 245-251.

Touitou, Y. (2001). Human aging and melatonin. clinical relevance. Experimental Gerontology 36, 1083-1100.

Vaiserman, A., A. Collinson, N. Koshel, I. Belaja, and V. Voitenko (2002, Dec). Seasonal programming of adult longevity in ukraine. Int J Biometeorol 47(1), 49-52.

Vaiserman, A. and V. Voitenko (2003). Early programming of adult longevity: demographic and experimental studies. J Anti Aging Med 6(1), 11-20.

Vaupel, J., K. Manton, and E. Stallard (1979). The impact of heterogeneity in individual frailty on the dynamics of mortality. Demography 16, 439-454. 
Wehr, T. (1997). Melatonin and seasonal rhythms. J Biol Rhythms 12, 518-527.

Wehr, T. (1998). Effect of seasonal changes in daylength on human neuroendocrine function. Hormone Research $49(118-124)$.

Yashin, A., K. Andreev, J. Curtsinger, and J. Vaupel (1996). Death-after-stress-data in the analysis of heterogeneous mortality. G. Christensen (Ed.), Transactions of Symposium in Applied Statistics, 24.

Yashin, A., J. Cypser, T. Johnson, A. Michalski, S. Boyko, and V. Novoseltsev (2002, March). Heat shock changes the heterogeneity distribution in populations of caenorhabditis elegans: does it tell us anything about the biological mechanism of stress response? The Journals of Gerontology. Series A, Biological Sciences and Medical Sciences 57(3), B83-B92.

Zapata, C., G. Alvarez, F. Rodriguez-Trelles, and X. Maside (2000, Oct). A long-term study on seasonal changes of gametic disequilibrium between allozymes and inversions in Drosophila subobscura. Evolution Int J Org Evolution 54(5), 1673-1679. 